\title{
Distributed Risk-Limiting Load Restoration in Unbalanced Distribution Systems with Networked Microgrids
}

Shen, Feifan; Wu, Qiuwei; Zhao, Jin; Wei, Wei; Hatziargyriou, Nikos D.; Liu, Feng

Published in:

IEEE Transactions on Smart Grid

Link to article, DOI:

10.1109/TSG.2020.2995099

Publication date:

2020

Document Version

Peer reviewed version

Link back to DTU Orbit

Citation $(A P A)$ :

Shen, F., Wu, Q., Zhao, J., Wei, W., Hatziargyriou, N. D., \& Liu, F. (2020). Distributed Risk-Limiting Load Restoration in Unbalanced Distribution Systems with Networked Microgrids. IEEE Transactions on Smart Grid, 11(6), 4574 - 4586. https://doi.org/10.1109/TSG.2020.2995099

\section{General rights}

Copyright and moral rights for the publications made accessible in the public portal are retained by the authors and/or other copyright owners and it is a condition of accessing publications that users recognise and abide by the legal requirements associated with these rights.

- Users may download and print one copy of any publication from the public portal for the purpose of private study or research.

- You may not further distribute the material or use it for any profit-making activity or commercial gain

- You may freely distribute the URL identifying the publication in the public portal 


\title{
Distributed Risk-Limiting Load Restoration in Unbalanced Distribution Systems with Networked Microgrids
}

\author{
Feifan Shen, Qiuwei Wu, Senior Member, IEEE, Jin Zhao, Student Member, IEEE, Wei Wei, Senior \\ Member, IEEE, Nikos D. Hatziargyriou, Fellow, IEEE, Feng Liu, Senior Member, IEEE
}

\begin{abstract}
To enhance the resilience of distribution systems (DSs), networked microgrids (MGs) can be employed to restore critical loads after a blackout. However, uncertainties of renewable energy sources (RESs) and loads bring challenges to load restoration. This paper proposes a distributed risk-limiting load restoration strategy for unbalanced DS with networked MGs. In the proposed strategy, the risk is represented by the conditional value-at-risk (CVaR), based on which a risk-limiting load restoration model is formulated considering renewable and load uncertainties. With the projection function-based alternating direction method of multipliers (P-ADMM) and sub-additivity of the CVaR, the proposed risk-limiting model is decoupled and solved in a distributed manner. This enables distributed risk limiting among MGs. Each MG can make load restoration decisions individually, while ensuring that the risk-limiting constraints of the overall system are satisfied. The modified IEEE 123-node system with networked MGs was used to conduct case studies. Simulation results show that the proposed strategy can restore loads efficiently with a smaller risk index and can provide distributed and flexible risk management. Moreover, the proposed distributed strategy can reduce the computation burden and is more robust against controller failures compared with the centralized strategy.
\end{abstract}

Index Terms-Alternating direction method of multipliers (ADMM), conditional value-at-risk (CVaR), distribution systems, distributed energy resources (DERs), load restoration, microgrids.

\section{INTRODUCTION}

$\mathrm{T}$ HE reliable operation of distribution systems (DSs) is constantly threatened by extreme weather events with increasing intensity [1]. To cope with this issue, resilience of DSs has become very important to withstand and recover from disruptions [2]. One key aspect of enhancing resilience is to implement automatic load restoration. After power outages or blackouts, the resilient DS uses distribution automation (DA)

This work was supported by the NTU-DTU joint PhD project of the Smart City joint program. Corresponding Author: Q.Wu (email: qw@elektro.dtu.dk)

F. Shen is with the Center for Electric Power and Energy, Department of Electrical Engineering, Technical University of Denmark, and also with the School of Electrical and Electronic Engineering, Nanyang Technological University, Singapore $639798 \quad$ (email: $\quad$ fshen@elektro.dtu.dk, feifan001@e.ntu.edu.sg).

Q. Wu is with the Center for Electric Power and Energy, Department of Electrical Engineering, Technical University of Denmark, Kgs. Lyngby, DK 2800 (email: qw@elektro.dtu.dk). devices, algorithms and communication technologies to restore loads automatically and intelligently [3].

Conventional load restoration strategies of DSs are mainly based on power fed by the upstream transmission systems (TSs) and, if needed, post-fault system reconfiguration. Various techniques have been used for conventional load restoration, such as expert systems [4], heuristic algorithms [5], multi-agent systems [6], meta-heuristic algorithms [7], and mathematical programming based on centralized [8], [9] and distributed optimization algorithms [2]. However, conventional load restoration strategies may be ineffective in case of damages in upstream TSs due to major disasters and unavailability of power from the upstream TSs for load restoration. Recently, utilizing microgrids (MGs) to deal with this challenge has been studied. A MG is a small-scale DS consisting of loads and different types of distributed energy resources (DERs) at low voltage (LV) or medium voltage (MV) level [1]. MGs can be used for load restoration in two ways: 1) MGs are considered as emergency sources to restore loads [10]-[11];2) the MG utilizes its local DERs to restore its own loads [12]-[16], or networked MGs are formed to restore loads together [17], which is the focus of this paper.

In [12], the formation of multiple MGs was proposed in the DS to restore loads. The restoration problem in [12] was formulated as a single time-step model without considering the restoration sequence. In [13], [14], sequential load restoration strategies were proposed to provide a series of control actions in MGs to coordinate DERs and switches to restore loads sequentially. In the above studies, uncertainties of load consumption and DERs generation, such as wind turbines (WTs) and photovoltaics (PVs), were not considered. However, uncertainties bring risks to the secure operation of the $\mathrm{MG}$ during restoration and thus they should be carefully addressed. A rolling-horizon optimization based stochastic load restoration

J. Zhao is with the Key Laboratory of Power System Intelligent Dispatch and Control of Ministry of Education, Shandong University, Jinan 250000, China (email: hizhaojin@163.com).

W. Wei and F. Liu are with the State Key Laboratory of Power Systems, Department of Electrical Engineering, Tsinghua University, Beijing 100084, China (Email: wei-wei04@mails.tsinghua.edu.cn, 1feng@tsinghua.edu.cn).

N. D. Hatziargyriou is with the School of Electrical and Computer Engineering, National Technical University of Athens (NTUA), 15773 Athens, Greece (e-mail: nh@power.ece.ntua.gr). 
strategy was proposed in [15] to sectionalize the outage portion of the DS into multiple MGs. In [16], a chance-constrained load restoration strategy was proposed, which generates the restoration sequence for MGs after blackouts. In [17], a risklimiting load restoration strategy was proposed for MGs using the risk-limiting dispatch method.

Most of the load restoration strategies of MGs are based on centralized optimization algorithms. A few challenges exist in these centralized load restoration strategies. Firstly, a central controller has to collect and process all the data required and send control commands, thus suffering from the risk of singlepoint failure and from a heavy computation burden. Secondly, since MGs may belong to different owners, they may not be willing to share all the privacy information during restoration [18], e.g., schedule schemes of DERs. Therefore, it is important to study distributed load restoration in networked MGs. The alternating direction method of multipliers (ADMM) [19] is a widely used distributed optimization algorithm, and relies on local data collection and information exchanges between neighboring agents. They are more resilient against failures and can improve computation efficiency exploiting parallel computation. In addition, the distributed optimization algorithm enables each MG to make decisions independently with limited boundary information exchanged.

The ADMM has been widely used for power system applications, such as the load restoration [2], optimal power flow (OPF) [20], [21], voltage control [22], and operation management of battery charging systems [23]. In [20], the convexified AC optimal power flow (OPF) problem was solved by the ADMM-based algorithm in a distributed manner at each node. In the algorithm, the closed-form solutions of subproblems can be derived, which significantly reduces computation time. The consensus-based ADMM was applied in [21] to solve the dynamic DC-OPF problem with demand response in a distributed manner. Based on the consensus-based ADMM, three distributed DC-OPF algorithms were proposed, including distributed DC-OPF with a central controller, fully decentralized DC-OPF, and distributed DC-OPF with the accelerated ADMM. They were compared with respect to convergence performance and communication requirements. In [22], a distributed voltage control scheme based on the ADMM was proposed for the large-scale wind farm cluster connected to the VSC-HDVC. Using the proposed distributed control scheme, the computation burden of the wind farm cluster and wind farm controllers and communication costs are reduced. The random-permuted ADMM was applied in [23] to solve the operation management problem of battery swapping-charging systems in a distributed way with less computation time. In [2], a distributed load restoration strategy based on the ADMM was proposed for distribution systems with coordinated operations of DERs. In this distributed strategy, the load restoration problem is formulated as a convex model with continuous variables and is solved in parallel at each node.

However, a number of control actions used for load restoration, such as operation of voltage regulators (VRs) and discontinuous load pick-up, can only be modeled as discrete variables. Therefore, the standard ADMM needs to be modified for non-convex problems of load restoration. A projection function-based ADMM (P-ADMM) was proposed in [24], [25] for the fast calculation of mixed integer programming (MIP) problems. In [26], the P-ADMM was applied to solve the coordinated dispatch problem of the PV inverter, on-load tap changer (OLTC) and capacitor banks, which is modeled as a mixed integer second-order cone programming (MISOCP) problem. It is shown that the P-ADMM is an effective method to solve non-convex problems with good convergence ability and a small optimality gap. Therefore, the P-ADMM is used in this study to solve the non-convex load restoration problem.

In addition, the conditional value-at-risk $(\mathrm{CVaR})$ is used to model distributed risk-limiting. The CVaR is a widely used risk-limiting index to deal with uncertainties, such as in optimal bidding of generation units [27], reactive power planning [28], unit commitment [29], and load restoration [30], [31]. Compared with the chance-constraint method, the CVaR has the sub-additivity feature, which makes it easier to realize the distributed risk limiting. In the CVaR-based formulation, the risk-limiting constraint can be directly decomposed among subsystems. Therefore, each sub-system can make risk-limiting decisions individually to realize the distributed risk limiting.

In [30], a CVaR-based load restoration strategy considering uncertainties of loads was proposed for TSs. The CVaR-based load restoration problem is formulated as a mixed-integer linear programming (MILP) model and solved by the particle swarm optimization (PSO) algorithm. In [31], a receding horizon load restoration strategy considering uncertainties of RESs and loads was proposed for coupled TSs and DSs based on the CVaR. The strategy is modeled as a two-stage optimization model with the relaxed multi-step optimization at the first stage and a singlestep tracing optimization at the second stage. With the receding horizon and $\mathrm{CVaR}$, this strategy can consider future conditions and achieve on-line restoration with the risk limited.

However, the CVaR-based load restoration strategies in [30], [31] are proposed for risk-limiting load restoration of TSs and DSs with power supply available from the TS. These strategies may be ineffective for load restoration of DSs in case of no power supply from the TS. Therefore, it is important to study the utilization of MGs for load restoration of DSs. In addition, in the above-mentioned CVaR-based studies [27]-[31], the CVaR-based risk-limiting constraints are incorporated into the optimization problem in a coupled way so the risk is limited in a centralized manner. To perform the distributed risk limit, this paper extends the CVaR-based risk-limiting constraints to a distributed form using the sub-additivity of the CVaR.

This paper proposes a distributed risk-limiting load restoration strategy for the unbalanced DS with networked MGs based on the CVaR and P-ADMM. In this strategy, the CVaR is used as a risk-limiting index, based on which the optimal risklimiting load restoration problem is formulated as a mixedinteger non-linear programming (MINLP) model, considering discrete variables and uncertainties of DERs and loads. Then, the MINLP model is transformed into a MILP model by applying linearization techniques. With the P-ADMM and subadditivity of the CVaR, the MILP model is solved in a distributed manner among MGs by iteratively solving small- 
scale quadratic programming (QP) sub-problems and applying projection functions to deal with discrete variables. Consequently, distributed risk limiting is realized among MGs, each of which can make load restoration decisions individually without violating risk-limiting constraints of the whole system.

The contributions of this paper are summarized as follows.

1) Develop a distributed risk-limiting load restoration strategy for unbalanced DSs with networked MGs, and formulate MINLP and MILP models for the CVaR-based optimal risk-limiting load restoration problem of the unbalanced DS with networked MGs;

2) Decompose the risk-limiting load restoration scheme by introducing the distributed risk limit using the subadditivity of the CVaR;

3) Develop a P-ADMM-based distributed solution method for the non-convex load restoration problem considering the discrete operation of VRs and load pick-up.

The rest of the paper is organized as follows: Section II describes the MINLP model of the optimal risk-limiting load restoration problem. Section III presents the formulation and decomposition of the MILP model. Section III presents the PADMM based distributed solution method for the MILP model. Case studies are provided in Section IV, followed by the conclusions.

\section{Optimal RisK-Limiting LoAd Restoration PROBLEM}

This section first introduces the load restoration scenario considered. The MINLP model of the optimal risk-limiting load restoration problem is formulated next.

\section{A. Load Restoration Scenario}

An unbalanced DS with three MGs shown in Fig. 1 is used to illustrate the load restoration scenario under consideration. The three MGs are connected by tie-lines and each MG consists of different types of DERs, i.e., micro-turbines (MTs), WTs, PVs and energy storage systems (ESSs). In the study, MTs and ESSs are dispatchable DERs and used to regulate frequency and voltage during the restoration process. The frequency and voltage regulation capabilities of the MT and ESS depend on their capacities. Moreover, the frequency regulation capability of the ESS is limited by its state of charge (SOC) level. The WTs and PVs are non-dispatchable DERs and their active power outputs are assumed to be the forecast values. Therefore, they do not participate in frequency regulation. Reactive power outputs of the WT and PV can be controlled to regulate voltage.

Suppose that the substation $(\mathrm{s} / \mathrm{s})$ is damaged due to extreme events and thus power from the upstream transmission system is not available for load restoration. During the outage period, networked MGs control DERs and conventional devices, i.e., VRs and capacitor banks, to pick up loads in a sequential procedure. During restoration, the frequency variation after a load pick-up is critical and requires special attention [32]. The risk of the frequency security violation due to uncertainties is limited by the CVaR-based method in this paper. It is assumed that there are sufficient resources to regulate voltage, such as VRs, capacitor banks and DERs, and the risk of voltage violation is not considered. Since this paper focuses on risk management of the frequency security, the uncertainties of DER generation and loads that significantly affect the active power balance of the system are considered. It is assumed that system parameters, e.g., line impedance, do not have uncertainty.

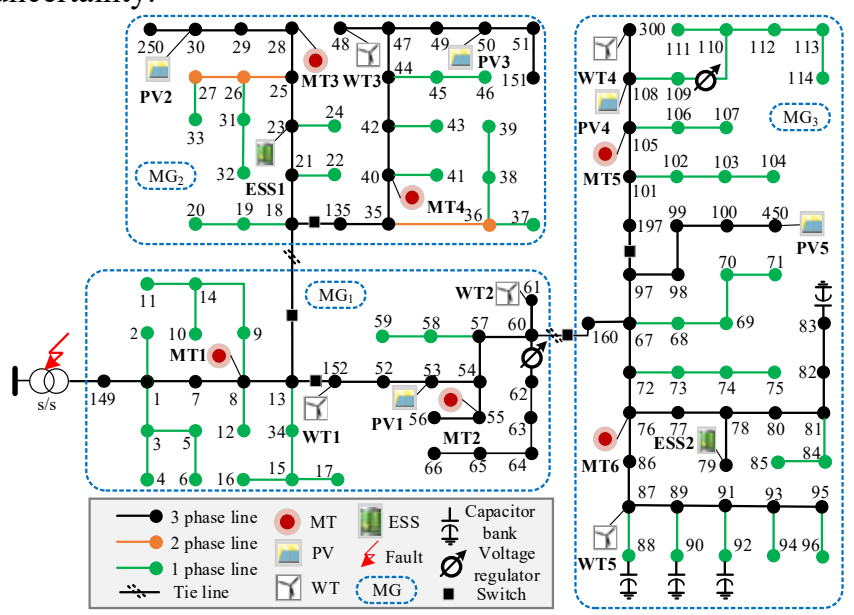

Fig. 1. The unbalance DS with networked MGs

\section{B. Optimal Risk-limiting Load Restoration Model}

Consider the DS as a directed graph $\mathcal{G}=(\mathcal{N}, \mathcal{L})$, where $\mathcal{N}$ denotes the set of nodes and $\mathcal{L}$ denotes the set of lines. Set $\mathcal{N}$ consists of $\mathcal{N}^{\mathrm{L}}, \mathcal{N}^{\mathrm{M}}, \mathcal{N}^{\mathrm{W}}, \mathcal{N}^{\mathrm{P}}, \mathcal{N}^{\mathrm{E}}$, and $\mathcal{N}^{\text {cap }}$, representing sets of loads, MTs, WTs, PVs, ESSs and capacitor banks, respectively. Set $\mathcal{L}$ consists of $\mathcal{L}^{\mathrm{N}}$ and $\mathcal{L}^{\mathrm{VR}}$, denoting sets of lines without VRs and lines with VRs, respectively. Set $\mathcal{T}$ is the set of restoration time steps. Set $\psi$ represents the three phases $\{a, b, c\} . \vec{x}_{i, t}$ and $\vec{x}_{i j, t}$ denote three-phase variables at node $i \in \mathcal{N}$ and line $i j \in \mathcal{L}$, respectively, at time step $t \in \mathcal{T} . \vec{x}(\phi)$ denotes the $\phi$-th element of $\vec{x}$. The inner and element-wise products of two vectors are denoted by $\langle\vec{x}, \vec{y}\rangle$ and $\vec{x} \odot \vec{y}$, respectively. The optimal risk-limiting load restoration problem is formulated as a MINLP model in (1)-(25) based on the steady state to decide the optimal restoration operation under uncertain conditions. To capture dynamics of MGs during restoration, a linearized frequency constraint is incorporated into the model to ensure the frequency stability of the obtained solution. The detailed dynamic analysis of frequency and voltage can be conducted using dynamic simulations before implementing the obtained restoration scheme.

\section{1) Objective function}

The objective function (1) consists of four terms. The first term maximizes the load pick-up amount considering priorities of loads, where $\vec{P}_{i, t}^{\mathrm{L}}$ represents the active load restored, $\vec{c}_{i}^{\mathrm{p}}$ is the priority coefficient, and $\Delta t$ is the duration of a time step. The second term minimizes active power losses, where $\vec{I}_{i j, t}^{\text {sqr }}$ is the square of the current magnitude flowing from node $i$ to node $j$, $\vec{R}_{i j}+j \vec{X}_{i j}$ is the impedance of line $i j$, and $\vec{c}_{i j}^{\mathrm{L}}$ is the cost 
coefficient. The third term minimizes MT generation costs, where $\vec{P}_{i, t}^{\mathrm{M}}$ is the MT active power generation and $\vec{c}_{i}^{\mathrm{M}}$ is the generation cost coefficient. The last term minimizes operation costs of VRs, where $\vec{S}_{i j, t}^{\mathrm{VR}}$ denotes the number of tap operations of the VR of line $i j$ and $\vec{c}_{i j}^{\mathrm{VR}}$ is the cost coefficient. The operation costs are considered in the objective function in order to minimize the number of operations of VRs. It is worth noting that minimizing the operation costs will not deteriorate load pick-up performance since the coefficients associated with the load pick-up amount are much larger than the ones of the operation costs.

$$
\begin{array}{r}
\max \sum_{i \in \mathcal{N}^{\mathrm{L}}} \sum_{t \in \mathcal{T}}\left\langle\vec{c}_{i}^{\mathrm{P}}, \vec{P}_{i, t}^{\mathrm{L}}\right\rangle \Delta t-\sum_{i j \in \mathcal{L}} \sum_{t \in \mathcal{T}}\left\langle\vec{c}_{i j}^{\mathrm{L}}, \vec{I}_{i j, t}^{\mathrm{sqr}} \odot \vec{R}_{i j}\right\rangle \Delta t \\
-\sum_{i \in \mathcal{N}^{\mathrm{M}}} \sum_{t \in \mathcal{T}}\left\langle\vec{c}_{i}^{\mathrm{M}}, \vec{P}_{i, t}^{\mathrm{M}}\right\rangle \Delta t-\sum_{i j \in \mathcal{L}^{\mathrm{VR}}} \sum_{t \in \mathcal{T}}\left\langle\vec{c}_{i j}^{\mathrm{VR}}, \vec{s}_{i j, t}^{\mathrm{VR}}\right\rangle
\end{array}
$$

\section{2) Load constraints}

The loads restored at node $i$ in time step $t\left(\vec{P}_{i, t}^{\mathrm{L}}+j \vec{Q}_{i, t}^{\mathrm{L}}\right)$ are modeled as,

$$
\begin{aligned}
& \vec{P}_{i, t}^{\mathrm{L}}=x_{i, t}^{\mathrm{L}} \vec{P}_{i, t}^{\mathrm{L}} ; \forall i \in \mathcal{N}^{\mathrm{L}}, t \in \mathcal{T} \\
& \vec{Q}_{i, t}^{\mathrm{L}}=x_{i, t}^{\mathrm{L}} \vec{Q}_{i, t}^{\mathrm{L}} ; \forall i \in \mathcal{N}^{\mathrm{L}}, t \in \mathcal{T}
\end{aligned}
$$

where $x_{i, t}^{\mathrm{L}}$ is the binary variable representing load pick-up status, $x_{i, t}^{\mathrm{L}}=1$ if the load is restored; otherwise, $x_{i, t}^{\mathrm{L}}=0 ; \vec{P}_{i, t}^{\mathrm{L}, \mathrm{F}}+j \vec{Q}_{i, t}^{\mathrm{L}, \mathrm{F}}$ represents the expected amount of loads. The cold load pick-up phenomenon (CLPU) is modeled by (3) [2], where $\vec{P}_{i, t}^{\text {CLPU }}$ denotes additional active power absorbed due to the CLPU, which is included in the right-hand side of (2a).

$$
\vec{P}_{i, t}^{\mathrm{CLPU}}=\beta_{i} \lambda_{i}\left(x_{i, t}^{\mathrm{L}}-x_{i, t-1}^{\mathrm{L}}\right) \vec{P}_{i, t}^{\mathrm{L}, \mathrm{F}} ; \forall i \in \mathcal{N}^{\mathrm{L}}, t \in \mathcal{T}
$$

where $\beta_{i}$ is the cold load percentage and $\lambda_{i}$ is the percentage of additional power absorbed. Note that adjustable loads can be modeled by defining $x_{i, t}^{\mathrm{L}}$ as the continuous variable.

\section{3) Voltage constraints}

The squared voltage magnitude $\left(\vec{V}_{i, t}^{\text {sqr }}\right)$ should be within the maximum and minimum limits $\left(\bar{V}^{\mathrm{sqr}}, \underline{V}^{\mathrm{sqr}}\right)$ as,

$$
\underline{V}^{\text {sar }} \leq \vec{V}_{i, t}^{\mathrm{sar}}(\phi) \leq \bar{V}^{\mathrm{sr}} ; \quad \forall i \in \mathcal{N}, t \in \mathcal{T}, \phi \in \psi
$$

\section{4) Line current capacity constraints}

The squared current magnitude ( $\vec{I}_{i j, t}^{\text {sqr }}$ ) should be lower than the maximum limit ( $\left.\bar{I}_{i j}^{\mathrm{sqr}}\right)$ as,

$$
\vec{I}_{i j, t}^{\mathrm{sqr}}(\phi) \leq \bar{I}_{i j}^{\mathrm{sar}} ; \quad \forall i j \in \mathcal{L}, t \in \mathcal{T}, \phi \in \psi
$$

\section{5) MT operation constraints}

The MT power generation $\left(\vec{P}_{i, t}^{\mathrm{MT}}+{ }_{j} \vec{Q}_{i, t}^{\mathrm{MT}}\right)$ is constrained by the maximum and minimum limits $\left(\vec{P}_{i}^{\mathrm{MT} \text { max }}, \vec{Q}^{\mathrm{MT} \text {,max }}, \vec{P}_{i}^{\mathrm{MT}, \mathrm{min}}\right.$, $\vec{Q}_{i}^{\mathrm{MT}, \mathrm{min}}$ ) in (6). Constraint (7) is the ramping rate constraint, where $\vec{P}_{i}^{\mathrm{MT}, \mathrm{ru}}$ and $\vec{P}_{i}^{\mathrm{MT}, \mathrm{rd}}$ are the ramp-up and ramp-down limits, respectively.

$$
\begin{aligned}
& \vec{P}_{i}^{\mathrm{MT}, \mathrm{min}} \leq \vec{P}_{i, t}^{\mathrm{MT}} \leq \vec{P}_{i}^{\mathrm{MT}, \mathrm{max}} ; \forall i \in \mathcal{N}^{\mathrm{M}}, t \in \mathcal{T} \\
& \vec{Q}_{i}^{\mathrm{MT}, \mathrm{min}} \leq \vec{Q}_{i, t}^{\mathrm{MT}} \leq \vec{Q}_{i}^{\mathrm{MT}, \mathrm{max}} ; \forall i \in \mathcal{N}^{\mathrm{M}}, t \in \mathcal{T} \\
& \vec{P}_{i}^{\mathrm{MT}, \mathrm{rd}} \leq \vec{P}_{i, t}^{\mathrm{MT}}-\vec{P}_{i, t-1}^{\mathrm{MT}} \leq \vec{P}_{i}^{\mathrm{MT}, \mathrm{ru}} ; \forall i \in \mathcal{N}^{\mathrm{M}},\left.t \in \mathcal{T}\right|_{t \neq t_{1}} \\
& \vec{P}_{i, t}^{\mathrm{MT}} \leq \vec{P}_{i}^{\mathrm{MT}, \mathrm{ru}} ; \quad \forall i \in \mathcal{N}^{\mathrm{M}}, t=t_{1}
\end{aligned}
$$

\section{6) WT and PV operation constraints}

The PV and WT active power generation $\left(\vec{P}_{i, t}^{\mathrm{PV}}, \vec{P}_{i, t}^{\mathrm{WT}}\right)$ is equal to their generation forecasts $\left(\vec{P}_{i, t}^{\mathrm{PV}, \mathrm{F}}, \vec{P}_{i, t}^{\mathrm{WT}, \mathrm{F}}\right)$ in (8). The PV and WT reactive power generation $\left(\vec{Q}_{i, t}^{\mathrm{PV}}, \vec{Q}_{i, t}^{\mathrm{WT}}\right)$ is constrained by the maximum and minimum limits $\left(\vec{Q}_{i}^{\mathrm{V}, \text { max }}, \vec{Q}_{i}^{\mathrm{p}, \text { min }}, \vec{Q}_{i, t}^{\mathrm{WT}, \mathrm{max}}\right.$ $\vec{Q}_{i, t}^{\mathrm{WT}, \mathrm{nin}}$ ) in (9).

$$
\begin{aligned}
& \vec{P}_{i, t}^{\mathrm{PV}}=\vec{P}_{i, t}^{\mathrm{PV}, \mathrm{F}} ; \forall i \in \mathcal{N}^{\mathrm{P}}, t \in \mathcal{T} \\
& \vec{P}_{i, t}^{\mathrm{WT}}=\vec{P}_{i, t}^{\mathrm{WT}, \mathrm{F}} ; \forall i \in \mathcal{N}^{\mathrm{W}}, t \in \mathcal{T} \\
& \vec{Q}_{i}^{\mathrm{PV}, \text { min }} \leq \vec{Q}_{i, t}^{\mathrm{PV}} \leq \vec{Q}_{i}^{\mathrm{PV}, \mathrm{max}} ; \forall i \in \mathcal{N}^{\mathrm{P}}, t \in \mathcal{T} \\
& \vec{Q}_{i, t}^{\mathrm{WT} \text { min }} \leq \vec{Q}_{i, t}^{\mathrm{WT}} \leq \vec{Q}_{i, t}^{\mathrm{WT}, \mathrm{max}} ; \forall i \in \mathcal{N}^{\mathrm{W}}, t \in \mathcal{T}
\end{aligned}
$$

\section{7) Energy storage system operation constraints}

Energy storage systems (ESSs) are modeled based on the per-phase basis [33] in (10)-(15). Constraint (10) calculates net active power generation $\left(\vec{P}_{i, t}^{\mathrm{ES}}\right.$ ) of the ESS, which is the difference between its discharging power $\left(\vec{P}_{i, t}^{\mathrm{ESS}, \mathrm{D}}\right)$ and charging power $\left(\vec{P}_{i, t}^{\mathrm{ESC}}\right)$.

$$
\vec{P}_{i, t}^{\mathrm{ES}}=\vec{P}_{i, t}^{\mathrm{ES}, \mathrm{D}}-\vec{P}_{i, t}^{\mathrm{ES}, \mathrm{C}} ; \forall i \in \mathcal{N}^{\mathrm{E}}, t \in \mathcal{T}
$$

Constraint (11) ensures that the ESS can only operate in one of the three modes, i.e., charging, discharging or idle mode.

$$
\vec{x}_{i, t}^{\mathrm{ESC}}(\phi)+\vec{x}_{i, t}^{\mathrm{ES}, \mathrm{D}}(\phi) \leq 1 ; \forall i \in \mathcal{N}^{\mathrm{E}}, t \in \mathcal{T}, \phi \in \psi
$$

where $\vec{x}_{i, t}^{\mathrm{ES}, \mathrm{C}}$ and $\vec{x}_{i, t}^{\mathrm{ESD}}$ are binary variables, $\vec{x}_{i, t}^{\mathrm{ESC}}=1$ if the ESS operates in the charging mode, $\vec{x}_{i, t}^{\mathrm{ES}, \mathrm{D}}=1$ if the ESS operates in the discharging mode, and both of them are zero if the ESS operates in the idle mode.

Constraints (12) and (13) are active and reactive power generation constraints of the ESS.

$$
\begin{aligned}
& \vec{x}_{i, t}^{\mathrm{ES}, \mathrm{C}} \odot \vec{P}_{i}^{\mathrm{EC}, \text { min }} \leq \vec{P}_{i, t}^{\mathrm{ES}, \mathrm{C}} \leq \vec{x}_{i, t}^{\mathrm{ES}, \mathrm{C}} \odot \vec{P}_{i}^{\mathrm{EC}, \text { max }} ; \forall i \in \mathcal{N}^{\mathrm{E}}, t \in \mathcal{T} \quad(12 \mathrm{a}) \\
& \vec{x}_{i, t}^{\mathrm{ES}, \mathrm{D}} \odot \vec{P}_{i}^{\mathrm{ED}, \text { min }} \leq \vec{P}_{i, t}^{\mathrm{ES} \mathrm{D}} \leq \vec{x}_{i, t}^{\mathrm{ES}, \mathrm{D}} \odot \vec{P}_{i}^{\mathrm{ED}, \text { max }} ; \forall i \in \mathcal{N}^{\mathrm{E}}, t \in \mathcal{T} \quad(12 \mathrm{~b}) \\
& \vec{x}_{i, t}^{\mathrm{ES}} \odot \vec{Q}_{i}^{\mathrm{E} \text { min }} \leq \vec{Q}_{i, t}^{\mathrm{ES}} \leq \vec{x}_{i, t}^{\mathrm{ES}} \odot \vec{Q}_{i}^{\mathrm{Emax}} ; \forall i \in \mathcal{N}^{\mathrm{E}}, t \in \mathcal{T}
\end{aligned}
$$


where $\vec{P}_{i}^{\mathrm{EC} \text { max }}$ and $\vec{P}_{i}^{\mathrm{EC} \text {,in }}$ are the maximum and minimum limits of active charging power, $\vec{P}_{i}^{\boxplus \max }$ and $\vec{P}_{i}^{\boxplus, \text { min }}$ are the maximum and minimum limits of active discharging power, $\vec{Q}_{i}^{\mathrm{ES}}$ is the reactive power output of the ESS, and $\vec{Q}_{i}^{\mathrm{Em} \text { mx }}$ and $\vec{Q}_{i}^{\mathrm{E} \text { min }}$ are the maximum and minimum limits of reactive power.

Constraint (14) represents the energy balance relation of the ESS, where $\vec{E}_{i, t}^{\mathrm{ES}}$ is the state of charge (SOC) of the ESS, $\vec{E}_{i, t}^{\mathrm{ES}, \text { ini }}$ is the initial SOC, $\eta_{i}^{\mathrm{C}}$ and $\eta_{i}^{\mathrm{D}}$ are charging and discharging efficiency coefficients, respectively.

$$
\begin{aligned}
\vec{E}_{i, t}^{\mathrm{ES}} & =\vec{E}_{i, t-1}^{\mathrm{ES}}+\eta_{i}^{\mathrm{C}} \vec{P}_{i, t}^{\mathrm{ES}, \mathrm{C}} \Delta t-\frac{1}{\eta_{i}^{\mathrm{D}}} \vec{P}_{i, t}^{\mathrm{ES}, \mathrm{D}} \Delta t ; \forall i \in \mathcal{N}^{\mathrm{E}},\left.t \in \mathcal{T}\right|_{t \neq t_{1}} \\
\vec{E}_{i, t}^{\mathrm{ES}} & =\vec{E}_{i, t}^{\mathrm{ES}, i n i} ; \forall i \in \mathcal{N}^{\mathrm{E}}, t=t_{1}
\end{aligned}
$$

Constraint (15) stipulates that the SOC should be within the maximum and minimum limits ( $\left.\vec{E}_{i, t}^{\mathrm{ES}, \max }, \vec{E}_{i, t}^{\mathrm{ES}, \mathrm{min}}\right)$.

$$
\vec{E}_{i, t}^{\mathrm{ES}, \min } \leq \vec{E}_{i, t}^{\mathrm{ES}} \leq \vec{E}_{i, t}^{\mathrm{ES}, \max } ; \forall i \in \mathcal{N}^{\mathrm{E}}, t \in \mathcal{T}
$$

respectively.

\section{8) Capacitor bank constraints}

The shunt capacitor bank is model in (16), where $\vec{Q}_{i}^{\mathrm{C}}$ is the capacitor capacity and $x_{i, t}^{\text {cp }}$ is the binary variable representing the switch on/off status. The capacitor switches on if $x_{i, t}^{\text {cp }}=1$, otherwise, the capacitor switches off.

$$
\vec{Q}_{i, t}^{\mathrm{cap}}=x_{i, t}^{\mathrm{cp}} \vec{Q}_{i}^{\mathrm{C}} ; \forall i \in \mathcal{N}^{\mathrm{cap}}, t \in \mathcal{T}
$$

9) Three-phase unbalanced power flow constraints

$$
\begin{gathered}
\vec{P}_{i, t}^{\mathrm{DER}}+\sum_{j i \in \mathcal{L}} \vec{P}_{j i, t}-\sum_{i j \in \mathcal{L}}\left(\vec{P}_{i j, t}+\mathbf{R}_{i j} \vec{I}_{i j, t}^{\mathrm{sqr}}\right)=\vec{P}_{i, t}^{\mathrm{L}} ; \forall i \in \mathcal{N}, t \in \mathcal{T} \\
\vec{Q}_{i, t}^{\mathrm{DER}}+\vec{Q}_{i, t}^{\mathrm{cap}}+\sum_{j i \in \mathcal{L}} \vec{Q}_{j i, t}-\sum_{i j \in \mathcal{L}}\left(\vec{Q}_{i j, t}+\mathbf{X}_{i j} \vec{I}_{i j, t}^{\mathrm{sqr}}\right)=\vec{Q}_{i, t}^{\mathrm{L}} ; \\
\forall i \in \mathcal{N}, t \in \mathcal{T} \\
\vec{V}_{i, t}^{\mathrm{sgr}}-\vec{V}_{j, t}^{\mathrm{sar}}=2\left(\tilde{\mathbf{R}}_{i j} \vec{P}_{i j, t}+\tilde{\mathbf{X}}_{i j} \vec{Q}_{i j, t}\right)+\left|\mathbf{Z}_{i j}\right|^{\ominus 2} \vec{I}_{i j, t}^{\mathrm{sqr}} ; \forall i j \in \mathcal{L}^{\mathrm{N}}, t \in \mathcal{T}
\end{gathered}
$$

$$
\vec{V}_{j, t}^{\mathrm{sqr}} \odot \vec{I}_{i j, t}^{\mathrm{sqr}}=\left(\vec{P}_{i j, t}\right)^{\odot 2}+\left(\vec{Q}_{i j, t}\right)^{\odot 2} ; \quad \forall i j \in \mathcal{L}, t \in \mathcal{T}
$$

Constraints (17) and (18) represent active and reactive power balance at node $i$ in time step $t$, respectively, where $\vec{P}_{i, t}^{\mathrm{DER}}+j$ $\vec{Q}_{i, t}^{\text {DER }}$ represents the total power generation of DERs, $\vec{P}_{i j, t}+j$ $\vec{Q}_{i j, t}$ represents power flow flowing from node $i$ to node $j$, $\mathbf{Z}_{i j}=\mathbf{R}_{i j}+j \mathbf{X}_{i j}$ is the three-phase line impedance matrix and $\mathbf{Z}_{i j} \in \mathbb{C}^{3 \times 3}$. Constraint (19) represents the voltage drop of line $i j$ in time step $t$, where $\tilde{\mathbf{R}}_{i j} \in \mathbb{C}^{3 \times 3}$ and $\tilde{\mathbf{X}}_{i j} \in \mathbb{C}^{3 \times 3}$ are equivalent three-phase resistance and reactance matrixes, respectively [2].
Constraint (20) defines the relation between the current magnitude and voltage magnitude.

10) Voltage regulator constraints

The VRs can be modeled by ideal three-phase transformers [2]. A line $i j$ with a VR can be divided into a line $i j^{*}$ containing a tap-changer, which is modeled in (21), and a line $j^{*} j$ having the same impedance as the line $i j$.

$$
\begin{aligned}
& \vec{V}_{i, t}^{\mathrm{sqr}}=\vec{a}_{i j, t}^{\odot 2} \odot \vec{V}_{j^{*}, t}^{\mathrm{sqr}} ; \forall i j \in \mathcal{L}^{\mathrm{VR}}, t \in \mathcal{T} \\
& \vec{a}_{i j, t}=\vec{a}_{i j, t}^{\min }+\Delta a_{i j, t} \vec{n}_{i j, t}^{\mathrm{tap}} ; \forall i j \in \mathcal{L}^{\mathrm{VR}}, t \in \mathcal{T} \\
& \vec{n}_{i j, t}^{\mathrm{tap}}(\phi) \in\left\{0,1, \ldots, n^{\max }\right\}, \forall \phi \in \psi
\end{aligned}
$$

where $\vec{a}_{i j, t}$ represents the voltage ratio, $\vec{n}_{i j, t}^{\text {tap }}$ is an integer variable representing the tap position of the tap-changer, $\Delta a_{i j, t}$ represents the change rate of the voltage ratio, and $\vec{a}_{i j, t}^{\min }$ is the lower bound of the voltage ratio. The number of tap operations is modeled in (22), where $\vec{n}_{i j}^{\text {tapini }}$ is the initial tap position.

$$
\begin{aligned}
& \vec{s}_{i j, t}^{\mathrm{VR}} \geq \vec{n}_{i j, t}^{\mathrm{tap}}-\vec{n}_{i j, t-1}^{\mathrm{tap}} ; \forall i j \in \mathcal{L}^{\mathrm{VR}},\left.t \in \mathcal{T}\right|_{t \neq t_{1}} \\
& \vec{s}_{i j, t}^{\mathrm{VR}} \geq \vec{n}_{i j, t-1}^{\mathrm{tap}}-\vec{n}_{i j, t}^{\mathrm{tap}} ; \forall i j \in \mathcal{L}^{\mathrm{VR}},\left.t \in \mathcal{T}\right|_{t \neq t_{1}} \\
& \vec{S}_{i j, t}^{\mathrm{VR}} \geq \vec{n}_{i j, t}^{\mathrm{tap}}-\vec{n}_{i j}^{\mathrm{tap}, \mathrm{ini}} ; \forall i j \in \mathcal{L}^{\mathrm{VR}}, t=t_{1} \\
& \vec{S}_{i j, t}^{\mathrm{VR}} \geq \vec{n}_{i j}^{\mathrm{tap}, \mathrm{ini}}-\vec{n}_{i j, t}^{\mathrm{tap}} ; \forall i j \in \mathcal{L}^{\mathrm{VR}}, t=t_{1}
\end{aligned}
$$

\section{1) Load pick-up sequencing constraints}

Constraint (23) represents that the load cannot be tripped once it has been restored.

$$
x_{i, t}^{\mathrm{L}} \geq x_{i, t-1}^{\mathrm{L}} ; \forall i \in \mathcal{N}^{\mathrm{L}}, t \in \mathcal{T}
$$

\section{2) Risk-limiting frequency response rate constraints}

The frequency response rate (FRR) is used to measure the frequency deviation after a sudden load pick-up, which can be modeled by a simplified inequality in (24) [16]. The FRR constraint denotes that the equivalent incremental load pick-up amount in each time step should be smaller than the maximum allowable amount that is proportional to the total capacity of all MTs and ESSs operating in discharging modes.

$$
\begin{aligned}
& \sum_{i \in \mathcal{N}^{\mathrm{L}}} \sum_{\phi \in \psi}\left(\Delta \vec{P}_{i, t}^{\mathrm{L}}(\phi)-\Delta \vec{P}_{i, t}^{\mathrm{PV}}(\phi)-\Delta \vec{P}_{i, t}^{\mathrm{WT}}(\phi)\right) \\
& \leq \gamma\left(\sum_{\phi \in \psi} \sum_{i \in \mathcal{N}^{\mathrm{M}}} \vec{P}_{i}^{\mathrm{MT}, \max }(\phi)+\sum_{i \in \mathcal{N}^{\mathrm{E}}}\left\langle\vec{x}_{i, t}^{\mathrm{ES}, \mathrm{D}}, \vec{P}_{i}^{\mathrm{ED}, \max }\right\rangle\right) ; \forall t \in \mathcal{T}
\end{aligned}
$$

where

$$
\left\{\begin{array}{l}
\Delta \vec{P}_{i, t}^{\mathrm{L}}=\vec{P}_{i, t}^{\mathrm{L}}-\vec{P}_{i, t-1}^{\mathrm{L}} ; \forall i \in \mathcal{N}^{\mathrm{L}}, t \in \mathcal{T} \\
\Delta \vec{P}_{i, t}^{\mathrm{PV}}=\vec{P}_{i, t}^{\mathrm{PV}, \mathrm{F}}-\vec{P}_{i, t-1}^{\mathrm{PV}, \mathrm{F}} ; \forall i \in \mathcal{N}^{\mathrm{P}}, t \in \mathcal{T} \\
\Delta \vec{P}_{i, t}^{\mathrm{WT}}=\vec{P}_{i, t}^{\mathrm{WT}, \mathrm{F}}-\vec{P}_{i, t-1}^{\mathrm{WT}, \mathrm{F}} ; \forall i \in \mathcal{N}^{\mathrm{W}}, t \in \mathcal{T}
\end{array}\right.
$$

For simplicity, the compact form of (24) is,

$$
R\left(\mathbf{x}^{\mathrm{L}}, \mathbf{y}\right) \leq R_{\mathrm{b}} ; \mathbf{x}^{\mathrm{L}}=\left[x_{i, t}^{\mathrm{L}}\right] ; \mathbf{y}=\left[\vec{P}_{i, t}^{\mathrm{LF}}, \vec{P}_{i, t}^{\mathrm{WT}, \mathrm{F}}, \vec{P}_{i, t}^{\mathrm{PV}, \mathrm{F}}\right]
$$

where function $R\left(\mathbf{x}^{\mathrm{L}}, \mathbf{y}\right)$ calculates the incremental load pick-up amount and $R_{\mathrm{b}}$ is the maximum allowable amount. In practice, since forecast errors are inevitable, the forecasts in $\mathbf{y}$ are 
uncertain variables $\mathbf{y}^{\mathrm{u}}$. Therefore, the risk of the FRR constraint violation caused by uncertainties should be limited. Based on the CVaR, the risk-limiting FRR constraint is formulated as,

$$
\operatorname{CVaR}\left(R\left(\mathbf{x}^{\mathrm{L}}, \mathbf{y}^{\mathrm{u}}\right)\right) \leq R_{\mathrm{b}} ; \mathbf{y}^{\mathrm{u}}=\left[\vec{P}_{i, t}^{\mathrm{L}, \mathrm{u}}, \vec{P}_{i, t}^{\mathrm{WT}, \mathrm{u}}, \vec{P}_{i, t}^{\mathrm{PV}, \mathrm{u}}\right]
$$

where $\vec{P}_{i, t}^{0, u}$ represents uncertain active loads and active power generation of WTs and PVs. Constraint (25) represents that the CVaR value of the uncertain incremental load pick-up amount $R\left(\mathbf{x}^{\mathrm{L}}, \mathbf{y}^{\mathrm{u}}\right)$ should be smaller than $R_{\mathrm{b}}$. By definition, the value-atrisk (VaR) value is the least value $\xi$ such that $R(\cdot)$ does not exceed the value $\xi$ with a specified probability level $\alpha$, whereas the $\mathrm{CVaR}$ value is the conditional expected value of $R(\cdot)$ above the value $\xi$. The $\mathrm{CVaR}$ value of $R\left(\mathbf{x}^{\mathrm{L}}, \mathbf{y}^{\mathrm{u}}\right)$ with the probability level $\alpha$ is calculated as,

$$
\operatorname{CVaR}\left(R\left(\mathbf{x}^{\mathrm{L}}, \mathbf{y}^{\mathrm{u}}\right)\right)=(1-\alpha)^{-1} \int_{R\left(\mathbf{x}^{\mathrm{L}}, \mathbf{y}^{\mathrm{u}}\right) \geq \xi} R\left(\mathbf{x}^{\mathrm{L}}, \mathbf{y}^{\mathrm{u}}\right) p\left(\mathbf{y}^{\mathrm{u}}\right) d \mathbf{y}^{\mathrm{u}}
$$

where $p\left(\mathbf{y}^{\mathrm{u}}\right)$ denotes the probability distribution of $\mathbf{y}^{\mathrm{u}}$.

Due to non-linear constraints (20), (21a), and (25), and integer variables, the optimal risk-limiting load restoration model is an MINLP model.

\section{Formulation AND DeCOMPOSITION OF THE MILP MODEL}

To make the optimal risk-limiting load restoration problem computationally tractable, the MINLP model is transformed into the MILP model by applying linearization techniques. Then, the decomposition of the MILP model among MGs is presented.

\section{A. Formulation of the MILP Model}

\section{1) Linearization of (20)}

The product of variables $\vec{V}_{j, t}^{\text {sqr }} \odot \vec{I}_{i j, t}^{\text {sqr }}$ in (20) is approximated by fixing $\vec{V}_{j, t}^{\text {sqr }}$ as the square of the nominal value $\left(V^{\text {nom }}\right)^{2}$. Since the voltage magnitude is constrained in a small range, the approximation error is low [8]. The square terms are approximated using the piecewise linearization technique [8] as shown in (26)-(27).

$$
\left(\vec{P}_{i j, t}\right)^{\odot 2} \approx h\left(\vec{P}_{i j, t}\right),\left(\vec{Q}_{i j, t}\right)^{\odot 2} \approx h\left(\vec{Q}_{i j, t}\right) ;
$$

where the function $h(z)$ is defined as,

$$
\begin{gathered}
h(z)=\sum_{\lambda=1}^{|\mathcal{P}|} \phi_{\lambda} \Delta z_{\lambda} ; \\
\sum_{\lambda=1}^{|\mathcal{P}|} \Delta z_{\lambda}=z^{+}+z^{-} ; z^{+}, z^{-} \geq 0 \\
z=z^{+}-z^{-} ; \\
0 \leq \Delta z_{\lambda} \leq \bar{z} /|\mathcal{P}|, \phi_{\lambda}=(2 \lambda-1) \bar{z} /|\mathcal{P}| ; \forall \lambda \in \mathcal{P}
\end{gathered}
$$

where $z$ is the argument of function $h, \mathcal{P}$ is the set of linearization segments of $z^{2},|\mathcal{P}|$ is the cardinality of $\mathcal{P}, z^{+}$and $z^{-}$are auxiliary variables used to calculate the absolute value of $z, \Delta z_{\lambda}$ is the auxiliary variable associated with the $\lambda$-th linearization segment whose slope is $\phi_{\lambda}$, and $\bar{z}$ is the maximum value of $z$. The linearization approximation of (20) is obtained as,

$$
\left(V^{\mathrm{nom}}\right)^{2} \vec{I}_{i j, t}^{\mathrm{sqr}}=h\left(\vec{P}_{i j, t}\right)+h\left(\vec{Q}_{i j, t}\right) ; \forall i j \in \mathcal{L}, t \in \mathcal{T}
$$

\section{2) Linearization of (21a)}

The binary expansion scheme [34] is used to linearize (21a). The integer variable $\vec{n}_{i j, t}^{\text {tap }}$ is represented by a binary equation in (29), where $\mathcal{H}$ is the set of binary representation and $\vec{\lambda}_{i j, t}^{h}$ is the binary variable.

$$
\vec{n}_{i j, t}^{\mathrm{tap}}=\sum_{h=1}^{|\mathcal{H}|} 2^{h-1} \vec{\lambda}_{i j, t}^{h}
$$

By introducing three auxiliary variables $\vec{m}_{i j, t}, \vec{e}_{i j, t}^{h}$ and $\vec{d}_{i j, t}^{h}$ , the constraint (21a) is linearized as (30), where $M$ is a large enough number.

$$
\begin{gathered}
\vec{V}_{i, t}^{\mathrm{sgr}}=a_{i j, t}^{\min } \vec{m}_{i j, t}+\Delta a_{i j, t} \sum_{h=1}^{H} 2^{h-1} \vec{d}_{i j, t}^{h} ; \\
\vec{m}_{i j, t}=\vec{a}_{i j, t} \vec{V}_{j^{\prime \prime}, t}^{\mathrm{sqr}}=a_{i j, t}^{\min } \vec{V}_{j^{*}, t}^{\mathrm{sr}}+\Delta a_{i j, t} \sum_{h=1}^{H} 2^{h-1} \vec{e}_{i j, t}^{h} ; \\
0 \leq \vec{e}_{i j, t}^{h} \leq M \vec{\lambda}_{i j, t}^{h}, 0 \leq \vec{d}_{i j, t}^{h} \leq M \vec{\lambda}_{i j, t}^{h} ; \\
0 \leq \vec{V}_{j^{*}, t}^{\mathrm{sqr}}-\vec{e}_{i j, t}^{h} \leq M\left(1-\vec{\lambda}_{i j, t}^{h}\right), 0 \leq \vec{m}_{i j, t}-\vec{d}_{i j, t}^{h} \leq M\left(1-\vec{\lambda}_{i j, t}^{h}\right)
\end{gathered}
$$

\section{3) Linearization of (25)}

According to [35], the CVaR value can be approximately calculated using (31) by discretizing uncertain variables $\mathbf{y}^{\mathrm{u}}$ into a collection of scenarios $\mathbf{y}_{j}^{\mathrm{u}}$, where $\mathcal{J}$ is the set of scenarios, $\pi_{j}$ is the probability of scenario $\mathbf{y}_{j}^{\mathrm{u}}$, and $[a]^{+}=\max \{a, 0\}$.

$$
\operatorname{CVaR}\left(R\left(\mathbf{x}, \mathbf{y}^{\mathrm{u}}\right)\right)=\xi+(1-\alpha)^{-1} \sum_{j=1}^{|\mathcal{J}|} \pi_{j}\left[R\left(\mathbf{x}, \mathbf{y}_{j}^{\mathrm{u}}\right)-\xi\right]^{+}
$$

Then, the non-linear constraint (25) can be replaced by linear equations in (32) by introducing the auxiliary variable $v_{j}[26]$.

$$
\begin{gathered}
\operatorname{CVaR}\left(R\left(\mathbf{x}, \mathbf{y}^{\mathrm{u}}\right)\right)=\xi+(1-\alpha)^{-1} \sum_{j=1}^{|J|} \pi_{j} v_{j} \leq R_{\mathrm{b}} ; \\
v_{j} \geq R\left(\mathbf{x}, \mathbf{y}_{j}^{\mathrm{u}}\right)-\xi, v_{j} \geq 0 ; j \in \mathcal{J}
\end{gathered}
$$

Finally, the MILP model is formulated by replacing nonlinear constraints (20), (21a) and (25) with linear constraints (28)-(30) and (32).

\section{B. Decomposition of the MILP model}

For the sake of presentation, define a set $\mathcal{K}_{i}:=\left\{a_{i}, a_{i j}\right\}$, which consists of all variables with the subscript of $i$ and $i j$ and represents exclusively affiliated variables of node $i$. According to the structure of networked MGs, the MILP model is rewritten in a compact form and decomposed as below,

$$
\min \sum_{\mathrm{MG}_{m} \in \mathcal{M}} f_{\mathrm{MG}_{m}}\left(\mathbf{x}_{\mathrm{MG}_{m}}^{\mathrm{b}}, \mathbf{x}_{\mathrm{MG}_{m}}^{\mathrm{c}}\right)
$$

subject to:

$$
g_{i j}\left(\vec{V}_{i}^{\text {sqr }}, \vec{V}_{j}^{\text {sqr }}, \vec{I}_{i j}^{\text {sqr }}, \vec{P}_{i j}, \vec{Q}_{i j}\right)=0 ; \forall i j \in \mathcal{L}^{\mathrm{T}}
$$




$$
\begin{array}{r}
g_{i}\left(\vec{P}_{j i}, \vec{Q}_{j i}, \vec{P}_{i j}, \vec{Q}_{i j}, \vec{I}_{i j}^{\mathrm{sqr}}\right)=0 ; \forall i \in \mathcal{B}_{m}, j \in \mathcal{B}_{n}, \mathrm{MG}_{m / n} \in \mathcal{M} \\
g_{\mathrm{MG}_{m}}\left(\mathbf{x}_{\mathrm{MG}_{m}}^{\mathrm{b}}, \mathbf{x}_{\mathrm{MG}_{m}}^{\mathrm{c}}, \vec{V}_{i}^{\mathrm{sqr}}, \vec{P}_{i j}, \vec{Q}_{i j}\right) \leq 0 ; \forall i j \in \mathcal{L}^{\mathrm{T}}, \mathrm{MG}_{m} \in \mathcal{M}
\end{array}
$$$$
\operatorname{CVaR}\left(\sum_{\mathrm{MG}_{m} \in \mathcal{M}} R_{\mathrm{MG}_{m}}\right) \leq R_{\mathrm{b}}
$$

The objective function in (33a) is the compact form of (1), where $\mathrm{MG}_{m} \in \mathcal{M}$ represents $m$-th $\mathrm{MG}, f_{\mathrm{MG}_{m}}$ is the objective function of $\mathrm{MG}_{m}, \quad \mathbf{x}_{\mathrm{MG}_{m}}^{\mathrm{b}}$ and $\mathbf{x}_{\mathrm{MG}_{m}}^{\mathrm{c}}$ represent binary and continuous variables of all nodes in $\mathrm{MG}_{m}$, respectively. Constraints (33b) and (33c) represent voltage drop constraints of tie lines $i j \in \mathcal{L}^{\mathrm{T}}$ and power balance constraints of the boundary node $i \in \mathcal{B}_{m}$ in $\mathrm{MG}_{m}$. As shown in (33b) and (33c), variables of boundary node $i \in \mathcal{B}_{m}$ in $\mathrm{MG}_{m}$, i.e., $\vec{V}_{i}^{\text {sqr }}, \vec{P}_{i j}, \vec{Q}_{i j}$, are coupled with variables of its adjacent boundary node $j \in \mathcal{B}_{n}$ in $\mathrm{MG}_{n}$, i.e., $\vec{V}_{j}^{\mathrm{sqr}}, \vec{P}_{j i}, \vec{Q}_{j i}$. Constraint (33d) represents local constraints of $\mathrm{MG}_{m}$ with exclusive variables. Inequality (33e) originates from the risk-limiting FRR constraint (25) which involves all MGs' situations, where uncertain variable $R_{\mathrm{MG}_{n}}$ represents the incremental load pickup amount in $\mathrm{MG}_{m}$.

To implement the distributed strategy, each MG should solve its own problem with exclusive variables. However, due to the coupled constraints (33b), (33c) and (33e), the MILP model (33) is not intrinsically separable with respect to MGs. Therefore, a P-ADMM based solution method is introduced in the next section to solve the MILP model in a distributed manner.

\section{P-ADMM BASED SOLUtion Method}

This section presents a P-ADMM based solution method to solve the MILP model in a distributed manner among MGs.

\section{A. Decoupling of Coupled Constraints}

Before solving the MILP model, the coupled constraints are decoupled by introducing auxiliary variables. For simplicity, the symbol of the three-phase variable $\vec{x}$ is simplified as $x$.

1) Decoupling of power flow constraints (33b) and (33c)

To decouple (33b) and (33c), a set of auxiliary variables and a set of equality constraints are introduced as,

$$
\left\{\begin{array}{l}
P_{j i}=\tilde{P}_{i j}, Q_{j i}=\tilde{Q}_{i j} ; \forall j i \in \mathcal{L}^{\mathrm{T}} \\
P_{i j}=\hat{P}_{i j}, Q_{i j}=\hat{Q}_{i j}, V_{i}^{\mathrm{sqr}}=\tilde{V}_{i}^{i, \mathrm{sqr}}, V_{j}^{\mathrm{sqr}}=\hat{V}_{i}^{j, \mathrm{sqr}} ; \forall i j \in \mathcal{L}^{\mathrm{T}}
\end{array}\right.
$$

By substituting auxiliary variables in (33b)-(33d), these constraints are transformed into (35), which can be fully decoupled among MGs, each of which optimizes its variables.

$$
\begin{aligned}
& g_{i j}\left(\tilde{V}_{i}^{i, \mathrm{sqr}}, \hat{V}_{i}^{j, \mathrm{sqr}}, I_{i j}^{\mathrm{sqr}}, \hat{P}_{i j}, \hat{Q}_{i j}\right)=0 ; \forall(i, j) \in \mathcal{L}^{\mathrm{T}} \\
& g_{i}\left(\tilde{P}_{i j}, \tilde{Q}_{i j}, \hat{P}_{i j}, \hat{Q}_{i j}, I_{i j}^{\mathrm{sgr}}\right)=0 ; \forall i \in \mathcal{B}_{m}, j \in \mathcal{B}_{n}, \mathrm{MG}_{m / n} \in \mathcal{M}(35 \mathrm{~b})
\end{aligned}
$$

$g_{\mathrm{MG}_{m}}\left(\mathbf{x}_{\mathrm{MG}_{m}}^{\mathrm{b}}, \mathbf{x}_{\mathrm{MG}_{m}}^{\mathrm{c}}, \tilde{V}_{i}^{i, \mathrm{sqr}}, \tilde{P}_{i j}, \tilde{Q}_{i j}\right) \leq 0 ; \forall i j \in \mathcal{L}^{\mathrm{T}}, \mathrm{MG}_{m} \in \mathcal{M}(35 \mathrm{c})$

2) Decoupling of the risk-limiting FRR constraint (33e)

The sub-additivity of the CVaR is shown in (36a), which means that the $\mathrm{CVaR}$ value of the overall system is smaller than the sum of CVaR values of all sub-systems. Therefore, as long as the right side of (36a) is smaller than the CVaR bound $R_{\mathrm{b}}$, as shown in (36b), the risk-limiting constraint (33e) is satisfied. Then, in order to have the distributed risk limit, the distributed constraint (36c) is introduced, where each sub-system limits its own $\mathrm{CVaR}$ value. It can be seen that, as long as the distributed constraint (36c) is satisfied, constraint (36b) is satisfied and thus the coupled constraint (33e) is satisfied. Therefore, the risk-limiting constraint (33e) is replaced by (36c). The solution obtained with the model using the distributed constraint (36c) respects the coupled risk-limiting constraint (33e).

$$
\begin{aligned}
& \operatorname{CVaR}\left(\sum_{\mathrm{MG}_{m} \in \mathcal{M}} R_{\mathrm{MG}_{m}}\right) \leq \sum_{\mathrm{MG}_{m} \in \mathcal{M}} \operatorname{CVaR}\left(R_{\mathrm{MG}_{m}}\right) ; \\
& \operatorname{CVaR}\left(\sum_{\mathrm{MG}_{m} \in \mathcal{M}} R_{\mathrm{MG}_{m}}\right) \leq \sum_{\mathrm{MG}_{m} \in \mathcal{M}} \operatorname{CVaR}\left(R_{\mathrm{MG}_{m}}\right) \leq R_{\mathrm{b}} ;
\end{aligned}
$$

$$
\left\{\begin{array}{l}
\operatorname{CVaR}\left(R_{\mathrm{MG}_{m}}\right) \leq R_{\mathrm{b}, \mathrm{MG}_{m}} ; \mathrm{MG}_{m} \in \mathcal{M} \\
R_{b, \mathrm{MG}_{m}}=\left(\sum_{\phi \in \Psi} \sum_{i \in \mathcal{N}_{\mathrm{MG}_{m}}} \vec{P}_{i}^{\mathrm{MT}, \max }(\phi)+\sum_{i \in \mathcal{N}_{\mathrm{MG}_{m}^{\mathrm{E}}}}\left\langle\vec{x}_{i, t}^{\mathrm{ESS},}, \vec{P}_{i}^{\mathrm{ED}, \max }\right\rangle\right) ; \\
\sum_{\mathrm{MG}_{m}} R_{\mathrm{b}^{\prime}, \mathrm{MG}_{m}}=R_{\mathrm{b}} ;
\end{array}\right.
$$

where $R_{\mathrm{b}, \mathrm{MG}_{m}}$ is the maximum allowable incremental load pick-up amount of $\mathrm{MG}_{m}$.

\section{3) Binary variables processing}

In the MILP model, all binary variables $\mathbf{x}_{\mathrm{MG}_{m}}^{\mathrm{b}}$ are redefined as continuous variables, and an equality constraint (37) and binary variables $\tilde{\mathbf{x}}_{\mathrm{MG}_{m}}^{\mathrm{b}}$ are introduced as,

$$
\mathbf{x}_{\mathrm{MG}_{m}}^{\mathrm{b}}=\tilde{\mathbf{x}}_{\mathrm{MG}_{m}}^{\mathrm{b}}, \mathbf{x}_{\mathrm{MG}_{m}}^{\mathrm{b}} \in[0,1], \tilde{\mathbf{x}}_{\mathrm{MG}_{m}}^{\mathrm{b}} \in\{0,1\} ; \forall \mathrm{MG}_{m} \in \mathcal{M}_{(37)}
$$

\section{B. Augmented Lagrangian}

By adding equality constraints (34) and (37) into the objective function (33a) through dual variables $\boldsymbol{\Lambda}$ defined in (38) and the penalty parameter $\rho$, the augmented Lagrangian of the MILP model is formulated, given by (39), subject to (35) and (36b). The augmented Lagrangian is optimized over primal variables $(\mathbf{X}, \mathbf{Y})$ defined in (40) and dual variables $\boldsymbol{\Lambda}$.

$$
\mathbf{\Lambda}=\left\{\chi_{i j}, \eta_{i j}, v_{i j}, \varsigma_{i j}, \kappa_{i}, \sigma_{i j}, \mathbf{o}_{\mathrm{MG}_{m}}\right\} ;
$$




$$
\begin{aligned}
& \min \sum_{\mathrm{MG}_{m} \in \mathcal{M}} f_{\mathrm{MG}_{m}}\left(\mathbf{x}_{\mathrm{MG}_{m}}^{\mathrm{b}}, \mathbf{A}_{\mathrm{MG}_{m}}\right)+\frac{\rho}{2}\left[\sum _ { j i \in \mathcal { L } ^ { \mathrm { T } } } \left(\left\|P_{j i}-\tilde{P}_{i j}+\chi_{i j}\right\|^{2}\right.\right. \\
& \left.+\left\|\chi_{i j}\right\|^{2}+\left\|Q_{j i}-\tilde{Q}_{i j}+\eta_{i j}\right\|^{2}-\left\|\eta_{i j}\right\|^{2}\right)+\sum_{i j \in \mathcal{L}^{\mathrm{T}}}\left(\left\|P_{i j}-\hat{P}_{i j}+v_{i j}\right\|^{2}\right. \\
& -\left\|v_{i j}\right\|^{2}+\left\|Q_{i j}-\hat{Q}_{i j}+\varsigma_{i j}\right\|^{2}-\left\|\sigma_{i j}\right\|^{2}+\left\|V_{i}^{\mathrm{sqr}}-\tilde{V}_{i}^{i, \mathrm{sqr}}+\kappa_{i}\right\|^{2} \\
& \left.-\left\|\kappa_{i}\right\|^{2}+\left\|V_{j}^{\mathrm{sqr}}-\hat{V}_{i}^{j, \mathrm{sqr}}+\sigma_{i j}\right\|^{2}-\left\|\sigma_{i j}\right\|^{2}\right)+\sum_{\mathrm{MG}_{m} \in \mathcal{M}}\left(\| \mathbf{x}_{\mathrm{MG}_{m}}^{\mathrm{b}}\right. \\
& \left.-\tilde{\mathbf{x}}_{\mathrm{MG}_{m}}^{\mathrm{b}}+\mathbf{o}_{\mathrm{MG}_{m}}\left\|^{2}-\right\| \mathbf{o}_{\mathrm{MG}_{m}} \|^{2}\right) ;
\end{aligned}
$$

subject to: (35) and (36b).

$$
\begin{aligned}
& \mathbf{X}=\left\{\tilde{V}_{i}^{i, \text { sqr }}, \hat{V}_{i}^{j, \text { sqr }}, \tilde{P}_{i j}, \tilde{Q}_{i j}, \hat{P}_{i j}, \hat{Q}_{i j}, \mathbf{x}_{\mathrm{MG}_{m}}^{\mathrm{b}}, \mathbf{x}_{\mathrm{MG}_{m}}^{\mathrm{c}}\right\} ; \\
& \mathbf{Y}=\left\{V_{i}, P_{i j}, Q_{i j}, \tilde{\mathbf{x}}_{\mathrm{MG}_{m}}^{\mathrm{b}}\right\} ;
\end{aligned}
$$

\section{P-ADMM-based algorithm}

The augmented Lagrangian is solved by the P-ADMM-based algorithm in an iterative procedure, as shown in Table I. For the ease of presentation, the objective function (39) of the augmented Lagrangian is represented by a compact form $L(\mathbf{X}$, $\mathbf{Y}, \boldsymbol{\Lambda})$.

TABLE I

PSEUD CODE OF THE P-ADMM BASED ALGORITHM

\begin{tabular}{l}
\hline P-ADMM based algorithm \\
\hline 1: Inputs: Parameters of the system and DERs, and forecast error \\
distributions \\
2: Output: load pick-up sequence, operations of DERs, VRs and \\
Capacitor banks, and power flow \\
3: while $\left\|\mathbf{X}^{\mathrm{ex},{ }^{*}, k}-\mathbf{Y}^{*}, k\right\|^{2}>\sigma_{1}$, or $\left\|\mathbf{X}^{\mathrm{ex},{ }^{*}, k}-\mathbf{X}^{\mathrm{ex},{ }^{*}, k-1}\right\|^{2}>\sigma_{2}$ \\
4: do \\
5: $\quad$ solve sub-problem I to update primal variables $\mathbf{X}$ \\
6: $\quad$ solve sub-problem II to update primal variables $\mathbf{Y}$ \\
7: update dual variables $\mathbf{\Lambda}$ using (44) \\
8: end
\end{tabular}

\section{1) Sub-problem I}

In the $k$-th iteration, primal variables $\mathbf{X}^{k}$ are optimized in subproblem I, given by (41), subject to (35) and (36b), with parameters $\mathbf{Y}^{*, k-1}$ and $\boldsymbol{\Lambda}^{*, k-1}$ obtained in the (k-1)-th iteration.

$$
\begin{gathered}
\text { Sub-problem I: } L\left(\mathbf{X}^{k}, \mathbf{Y}^{*, k-1}, \boldsymbol{\Lambda}^{*, k-1}\right) \\
\text { s.t. (35) and (36b) }
\end{gathered}
$$

Sub-problem I is a QP problem whose objective function and constraints can be fully decoupled among MGs. Therefore, subproblem I can be solved in a distributed manner among MGs.

\section{2) Sub-problem II}

The primal variables $\mathbf{Y}^{k}$ are optimized in sub-problem II, given by (42), with parameters $\mathbf{X}^{*, k-1}$ and $\boldsymbol{\Lambda}^{*, k-1}$.

$$
\text { Sub-problem II: } L\left(\mathbf{X}^{*, k-1}, \mathbf{Y}^{k}, \boldsymbol{\Lambda}^{*, k-1}\right)
$$

Sub-problem II is a non-constrained mixed integer quadratic programming (MIQP) problem. Instead of solving the MIQP problem (42) to optimize binary variables, the binary variables are optimized using the projection function in (43), where $\mathbf{P}_{x}$ denotes the projection function, which rounds each entry to its nearest binary value. Therefore, the last term in (39) associated with binary variables is excluded in sub-problem II and primal variables $\mathbf{Y}^{k}$ are optimized by solving a QP problem, which can be fully decoupled among MGs.

$$
\tilde{\mathbf{x}}_{\mathrm{MG}_{m}}^{\mathrm{b}, k}=\mathbf{P}_{x}\left(\mathbf{x}_{\mathrm{MG}_{m}}^{\mathrm{b}, * k-1}+\mathbf{o}_{\mathrm{MG}_{m}}^{*, k-1}\right) ;
$$

\section{3) Update of dual variables}

After optimizing primal variables, dual variables are updated in (44a) using the primal variables $\mathbf{Y}$ and exchanged variables $\mathbf{X}^{\mathrm{ex}}$ defined in $(44 \mathrm{~b})$,

$$
\begin{aligned}
\boldsymbol{\Lambda}^{k} & =\boldsymbol{\Lambda}^{*, k-1}+\left(\mathbf{X}^{\mathrm{ex}, *, k-1}-\mathbf{Y}^{*}, k\right) \\
\mathbf{X}^{\mathrm{ex}} & =\left\{\tilde{V}_{i}^{i, \mathrm{sqr}}, \hat{V}_{i}^{j, \mathrm{sqr}}, \tilde{P}_{i j}, \tilde{Q}_{i j}, \hat{P}_{i j}, \hat{Q}_{i j}, \mathbf{x}_{\mathrm{MG}_{m}}^{\mathrm{b}}\right\} ;
\end{aligned}
$$

\section{4) Stop criteria}

The optimization of primal variables and update of dual variable continue iteratively till primal and dual residuals are lower than specified thresholds, respectively.

$$
\left\|\mathbf{X}^{\mathrm{ex},{ }^{,}, k}-\mathbf{Y}^{*, k}\right\|^{2} \leq \sigma_{1},\left\|\mathbf{X}^{\mathrm{ex},{ }^{*}, k}-\mathbf{X}^{\mathrm{ex},{ }^{*}, k-1}\right\|^{2} \leq \sigma_{2},
$$

\section{CASE Studies}

The modified IEEE 123-node system with three MGs in Fig. 1 is used to demonstrate the effectiveness of the proposed distributed risk-limiting load restoration strategy. The detailed parameters of the system can be found in [36]. The DERs considered are categorized in Table II with respect to their black start and dispatchable capabilities. The MT at node 55 operates with the VSI mode and provides the reference voltage for the system [32]. The restoration horizon has six steps and each step has a duration of 15 minutes. The lower and upper limits of the voltage magnitude are 0.95 p.u. and 1.05 p.u., respectively. The probability level $\alpha$ is 0.9 . In the P-ADMM-based algorithm, convergence thresholds $\sigma_{1}$ and $\sigma_{2}$ are set as $10^{-4} \sqrt{|\mathcal{M}|}$ [2]. The coefficients in the objective function are monetized. The costs of the MT generation, line loss and operation of the VR at a time are set as $0.1 \mathrm{DKK} / \mathrm{kWh}, 0.1 \mathrm{DKK} / \mathrm{kWh}$, and $0.01 \mathrm{DKK}$, respectively. To ensure that the minimization of operation costs will not compromise the maximization of the load pick-up amount, the priority coefficients of the critical and non-critical loads are set as $1.5 \mathrm{DKK} / \mathrm{kWh}$ and $1.0 \mathrm{DKK} / \mathrm{kWh}$, respectively.

\begin{tabular}{|c|c|c|c|}
\hline DERs & & locations (node no.) & $\begin{array}{l}\text { black-start } \\
\text { capability }\end{array}$ \\
\hline dispatchable & MTs & $8,55,28,40,105,76$ & 55 \\
\hline
\end{tabular}

When applying the CVaR method to the case with multiple random variables, i.e., forecast errors of the WT and PV active power outputs and loads, the correlations among random variables should be considered when generating scenarios. In the study, the forecast error scenarios considering correlations are obtained using the copula function-based scenario generation method in [37], [38]. 1000 scenarios are generated and reduced to 20 scenarios by using the simultaneous backward reduction method [39]. The proposed algorithm is implemented using GAMS and the solution is obtained with the CPLEX solver.

TABLE II

CATEGORY OF DistRIBUTED ENERGY RESOURCES (DERS) 


\begin{tabular}{cc|c|c}
\hline \hline & ESSs & 23,79 & - \\
\hline \multirow{2}{*}{ non-dispatchable } & WTs & $152,61,48,300,87$ & - \\
& PVs & $53,30,50,108,450$ & - \\
\hline \hline
\end{tabular}

\section{A. Convergence Analysis}

Although the convergence of the P-ADMM-based algorithm cannot be guaranteed for the proposed MILP problem, the algorithm shows good performance to handle binary variables and converge to an acceptable solution for the MILP problems [24], [25] and MISOCP problems [26]. A convergence analysis is conducted by implementing the algorithm with different values of $\rho$ between 15 and 500. Table III shows that the algorithm can reach convergence within 700 iterations for different values of $\rho$. Moreover, the algorithm can converge to the optimal solution of the Augmented Lagrangian in (35), (36b), and (39), with these values of $\rho$, which shows a good convergence ability of the algorithm. In addition, the convergence performance of the algorithm highly depends on the selection of $\rho$. For instance, an improper value $\rho=500$ would cause slow convergence. Within the certain range, the algorithm with $\rho=30$ has the best convergence performance. The convergence process of the P-ADMM-based algorithm with $\rho=30$ is shown in Fig. 2. It can be seen that the primal and dual residuals are lower than thresholds after 182 iterations, which further demonstrates the feasibility and convergence of the proposed algorithm.

TABLE III

NUMBERS OF ITERATIONS WITH DIFFERENT VALUES OF $\rho$

\begin{tabular}{c|ccccccc}
\hline \hline$\rho$ & 15 & 20 & $\mathbf{3 0}$ & 40 & 50 & 100 & 150 \\
\hline$\#$ & 237 & 187 & $\mathbf{1 8 2}$ & 166 & 257 & 500 & 508 \\
\hline \hline$\rho$ & 200 & 250 & 300 & 350 & 400 & 450 & 500 \\
\hline$\#$ & 619 & 604 & 599 & 655 & 671 & 651 & 617 \\
\hline \hline
\end{tabular}

\#: the number of iterations

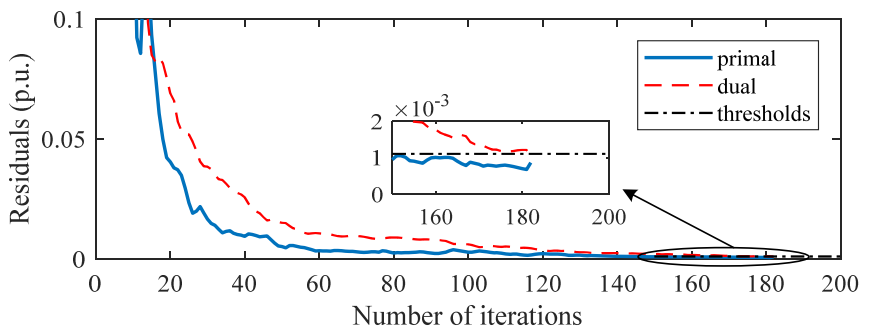

Fig. 2. Convergence process of the proposed algorithm $(\rho=30)$

\section{B. Risk-Limiting Load Restoration Performance}

\section{1) Restoration procedure}

Three methods are conducted to demonstrate the effectiveness of the proposed distributed risk-limiting strategy. The three methods are: 1) the optimal load restoration without considering risk limitation (OLR); 2) the centralized risklimiting load restoration (CRLR); 3) the proposed distributed risk-limiting load restoration (DRLR). The accumulated percentages of the load pick-up amount (load pick-up results), risk indexes $R_{\text {index }}$ and CVaR values with the three methods are listed in Table IV. The load pick-up results have been validated by conducting the power flow analysis based on the expected forecast values. 1000 scenarios randomly generated by the Monte Carlo simulation are used to check the risk index. The risk index at each restoration step denotes the percentage of those scenarios in which the FRR constraints are violated among the 1000 scenarios.

TABLE IV

LOAD RESTORATION RESULTS OF THREE METHODS

\begin{tabular}{cccccccc}
\hline \hline \multirow{2}{*}{ index } & \multirow{2}{*}{ method } & \multicolumn{7}{c}{ time steps } & \multirow{2}{*}{\begin{tabular}{c} 
obj. \\
\cline { 2 - 8 }
\end{tabular}} & & $t_{1}$ & $t_{2}$ & $t_{3}$ & $t_{4}$ & $t_{5}$ & value \\
\hline \multirow{2}{*}{$\begin{array}{c}\text { load } \\
\text { pick- } \\
\text { up }\end{array}$} & OLR & $32.94 \%$ & $56.0 \%$ & $77.4 \%$ & $100 \%$ & $100 \%$ & 306.40 \\
\cline { 2 - 8 } & CRLR & $29.0 \%$ & $54.4 \%$ & $73.9 \%$ & $93.2 \%$ & $100 \%$ & 297.43 \\
\cline { 2 - 8 } & DRLR & $27.8 \%$ & $50.2 \%$ & $70.2 \%$ & $88.8 \%$ & $100 \%$ & 288.16 \\
\hline \multirow{3}{*}{$R_{\text {index }}$} & OLR & $32.7 \%$ & $49.4 \%$ & $47.8 \%$ & $47.6 \%$ & $100 \%$ & $/$ \\
\cline { 2 - 8 } & CRLR & $3.6 \%$ & $6.2 \%$ & $20.3 \%$ & $6.4 \%$ & 0 & $/$ \\
\cline { 2 - 8 } & DRLR & $0.4 \%$ & $0.7 \%$ & $5.5 \%$ & $0.8 \%$ & 0 & $/$ \\
\hline \multirow{3}{*}{ CVaR } & OLR & 66.1 & 68.2 & 65.1 & 69.2 & -12.3 & 6.0 \\
\cline { 2 - 8 } & CRLR & 62.3 & 62.2 & 61.8 & 61.8 & 13.6 & 0.7 \\
\cline { 2 - 8 } & DRLR & 59.6 & 57.2 & 58.2 & 56.5 & 32.6 & -1.9 \\
\hline \multirow{2}{*}{$R_{\mathrm{b}}$} & CRLR & 62.4 & 62.4 & 62.4 & 62.4 & 60.0 & 61.2 \\
\cline { 2 - 8 } & DRLR & 62.4 & 62.4 & 62.4 & 61.2 & 61.2 & 60.0 \\
\hline \hline
\end{tabular}

As shown in Table IV, all loads can be restored during the restoration horizon in three methods. Although the CRLR and DRLR strategies pick up loads slowly and have lower objective values compared with the OLR strategy, the risk indexes are reduced substantially. For example, the CRLR and DRLR strategies pick up fewer loads in step $t_{1}$ to reduce the risk of the FRR constraint violation. Moreover, the DRLR strategy picks up fewest loads and consequently has the smallest $C V$ aR value and risk index. In addition, Table IV shows that the CVaR values of the DRLR strategy are lower than its bounds $\left(R_{\mathrm{b}}\right)$ in each step. This means that the risk-limiting constraints of the whole system are satisfied as long as the risk-limiting constraints of each MG are satisfied. The above results demonstrate that the proposed DRLR strategy can restore loads efficiently with a smaller risk index and has the distributed risklimiting ability.

Furthermore, two scenarios are conducted to study the impacts of VRs and CLPU phenomenon on load restoration. Load pick-up results in two scenarios are listed in Table V. In scenario 1, the operation of VRs is not considered. Compared with the base scenario considering the operation of VRs and CLPU phenomenon, loads are picked up slowly in scenario 1 and only $95.6 \%$ of loads are restored at the end of the restoration horizon, even if additional four restoration steps are included, i.e., a horizon with 10 restoration steps. This is because the loads at node 66 remain de-energized to avoid the voltage magnitude violation at node 64 at phase $b$. Thus, using VRs can facilitate load restoration. In scenario 2, the CLPU phenomenon is not considered. The results show that the restoration procedure picks up loads more rapidly and has a larger objective value. It means that the CLPU phenomenon defers the restoration procedure because additional power is absorbed due to the CLPU.

TABLE V

RESTORATION RESULTS IN TWO SCENARIOS

\begin{tabular}{|c|c|c|c|c|c|c|c|}
\hline \multicolumn{7}{|c|}{ time steps } & \multirow{2}{*}{$\begin{array}{c}\text { obj. } \\
\text { value }\end{array}$} \\
\hline & $t_{1}$ & $t_{2}$ & $t_{3}$ & $t_{4}$ & $t_{5}$ & $t_{6}$ & \\
\hline base & $27.8 \%$ & $50.2 \%$ & $70.2 \%$ & $88.8 \%$ & $100 \%$ & $100 \%$ & 288.16 \\
\hline sce 1 & $27.8 \%$ & $50.2 \%$ & $70.2 \%$ & $88.5 \%$ & $95.6 \%$ & $95.6 \%$ & 283.29 \\
\hline sce2 & $35.9 \%$ & $55.7 \%$ & $75.8 \%$ & $91.1 \%$ & $100 \%$ & $100 \%$ & 302.29 \\
\hline
\end{tabular}

2) Flexible risk management

The proposed DRLR strategy keeps the flexible risk management ability of the CVaR method. Table VI shows load 
pick-up results with different probability levels $\alpha$. It is shown that a larger probability level leads to a more conservative restoration solution with a slower load pick-up sequence and a smaller risk index. Therefore, flexible risk management can be realized by adjusting the probability level.

TABLE VI

LOAD PICK-UP RESULTS WITH DIFFERENT PROBABILITY LEVELS

\begin{tabular}{cccccccc}
\hline \multirow{2}{*}{$\alpha$} & \multicolumn{5}{c}{ time steps } & obj. & $R_{\text {index }}$ \\
\cline { 2 - 6 } & $t_{1}$ & $t_{2}$ & $t_{3}$ & $t_{4}$ & $t_{5}$ & value & in total \\
\hline 0.70 & $28.1 \%$ & $51.7 \%$ & $72.2 \%$ & $91.7 \%$ & $100 \%$ & 291.87 & $16.6 \%$ \\
0.80 & $28.1 \%$ & $50.5 \%$ & $71.8 \%$ & $90.7 \%$ & $100 \%$ & 290.33 & $12.9 \%$ \\
0.95 & $28.3 \%$ & $49.0 \%$ & $69.6 \%$ & $87.9 \%$ & $100 \%$ & 287.00 & $5.8 \%$ \\
\hline
\end{tabular}

\section{Computation Efficiency of the Distributed Strategy}

The computation time of the DRLR strategy is 355.3 seconds, which is lower than 455.2 seconds of the CRLR strategy. As far as the communication delay of the distributed method is concerned, this study assumes the synchronous setting of the P-ADMM algorithm, which means that all subproblems need to be solved before starting the next iteration [40] no matter how much time the communication delay is. Therefore, the communication delay would not affect the effectiveness of the proposed algorithm but increase the total execution time (computation time plus the total communication delay). Since the system of networked MGs is not big and suppose that there are direct fiber optical links between neighboring controllers, the communication delay is small and assumed to be 1 second.

Regarding the model size, the CRLR strategy solves an MILP problem with 93195 continuous variables and 648 binary variables, whereas the DRLR strategy solves smaller-scale QP problems, each of which has in average 16335 continuous variables in each calculation. The binary variables are dealt with by the projection function and not included in the optimization model. Therefore, the DRLR strategy outperforms the CRLR strategy in term of computation efficiency.

\section{Faults on Lines, Controllers or Communication Links}

\section{1) Scenario 3: Faults on lines}

Suppose that there is a fault on the line between nodes 35 and 135 within $\mathrm{MG}_{2}$ during the restoration horizon. Therefore, the switch between nodes 18 and 135 is opened to isolate the fault and nodes 35-51, 135 and 151 should remain de-energized during restoration. The load pick-up result is shown in Table VII. It can be seen that the remaining $79.5 \%$ of loads can be fully restored after 5 restoration steps.

\section{2) Scenario 4: Faults on restoration controllers}

Compared with the centralized strategy, the distributed strategy does not have the risk of the single point of failure. If one controller fails, the remaining controllers will still work. It is assumed that the restoration controller of $\mathrm{MG}_{2}$ has a fault and thus does not participate in the restoration process. In such a case, the tie-switch between nodes 13 and 18 is opened and the loads in $\mathrm{MG}_{2}$ remain de-energized. The load pick-up result with the proposed distributed scheme is shown in Table VII. It can be seen that all loads in $\mathrm{MG}_{1}$ and $\mathrm{MG}_{3}(68.8 \%$ of loads) can be restored during the restoration horizon. In the centralized strategy, if the central controller fails, the restoration process cannot be started.

\section{3) Scenario 5: Faults on communication links}

Suppose that faults occur on communication links between restoration controllers. In such a case, all MGs are disconnected by opening tie-switches and each controller makes restoration decisions based on local information. The load pick-up result is shown in Table VII. Since MGs are disconnected, there are no power exchanges between MGs during the restoration process. Therefore, it has the slower restoration procedure when all MGs are disconnected.

TABLE VII

RESTORATION RESULTS WITH THREE FAULT CONDITIONS

\begin{tabular}{|c|c|c|c|c|c|c|c|}
\hline & \multicolumn{6}{|c|}{ time steps } & \multirow{2}{*}{$\begin{array}{c}\text { obj. } \\
\text { value }\end{array}$} \\
\hline & $t_{1}$ & $t_{2}$ & $t_{3}$ & $t_{4}$ & $t_{5}$ & t6 & \\
\hline sce3 & $27.9 \%$ & $44.3 \%$ & $56.4 \%$ & $68.8 \%$ & $79.5 \%$ & $79.5 \%$ & 214.3 \\
\hline sce 4 & $19.1 \%$ & $33.5 \%$ & $45.2 \%$ & $58.2 \%$ & $68.8 \%$ & $68.8 \%$ & 178.2 \\
\hline $\operatorname{sce} 5$ & $27.8 \%$ & $50.7 \%$ & $69.6 \%$ & $88.2 \%$ & $100 \%$ & $100 \%$ & 286.7 \\
\hline
\end{tabular}

\section{E. Impact of Number of Reduced Scenarios on Solutions}

Table VIII shows the load pick-up results with different numbers of reduced scenarios, i.e., 4, 20 and 50 scenarios. It is shown that it has a more conservative solution with a lower risk index as the number of reduced scenarios increases. This is expected because the uncertainty can be captured more accurately with more scenarios considered in the model. However, the number of scenarios affects the computation complexity of the problem and thus affects convergence performance of the algorithm. Compared with the solution with 4 scenarios, the risk index of the solution with 20 scenarios decreases by 0.079 and computation time increases by 1.68 times. Although the risk index can be further decreased by 0.046 when 50 scenarios are used, the computation time increases by 1.35 times. Therefore, 20 scenarios are chosen in the study because it has a good tradeoff between the computation complexity and modeling accuracy of the uncertainty.

TABLE VIII

LOAD PICK-UP RESUltS WITH DIFFERENT REDUCED SCENARIOS WITH THE ALGORITHM WITH $\rho=30$

\begin{tabular}{|c|c|c|c|c|c|c|c|}
\hline \multirow{2}{*}{$\#$} & \multicolumn{5}{|c|}{ time steps } & \multirow{2}{*}{$\begin{array}{c}\text { compu. } \\
\text { time }\end{array}$} & \multirow{2}{*}{$\begin{array}{c}R_{\text {index }} \\
\text { in total }\end{array}$} \\
\hline & $t_{1}$ & $t_{2}$ & $t_{3}$ & $t_{4}$ & $t_{5}$ & & \\
\hline 4 & $27.4 \%$ & $49.0 \%$ & $68.8 \%$ & $88.8 \%$ & $100 \%$ & $254.8 \mathrm{~s}$ & $15.3 \%$ \\
\hline 20 & $27.8 \%$ & $50.2 \%$ & $70.2 \%$ & $88.8 \%$ & $100 \%$ & $428.7 \mathrm{~s}$ & $7.4 \%$ \\
\hline 50 & $27.1 \%$ & $48.7 \%$ & $68.2 \%$ & $87.1 \%$ & $100 \%$ & $581.7 \mathrm{~s}$ & $2.8 \%$ \\
\hline
\end{tabular}

\#: the number of reduced scenarios

\section{F. Impact of Restoration Horizon on Computation Complexity}

The computation complexity of the problem is summarized in Table IX with different numbers of restoration steps, i.e., 6, 8 and 10 steps. The numbers of variables and constraints of the problem and computation time are listed. It is shown that the problem considering a longer restoration horizon has more variables and constraints and needs more computation time. Since all loads can be restored after 5 restoration steps, as shown in Table IV, the restoration horizon with 6 restoration steps is chosen in this study.

TABLE IX

COMPUTATION COMPLEXITY OF THE PROBLEM With DifFERENT RESTORATION HORIZONS

\begin{tabular}{c|ccc}
\hline \hline $\begin{array}{c}\text { number of } \\
\text { restoration steps }\end{array}$ & 6 & 8 & 10 \\
\hline \hline
\end{tabular}




\begin{tabular}{c|c|ccc}
\hline \hline \multirow{2}{*}{ variables } & $\begin{array}{c}\text { binary } \\
\text { continuous }\end{array}$ & 648 & 864 & 1080 \\
& 93195 & 124602 & 155729 \\
\hline \multicolumn{2}{c}{ constraints } & 10716 & 14669 & 18342 \\
\hline \multicolumn{2}{c}{ computation time (s) } & 455.3 & 633.9 & 1333.1 \\
\hline \hline
\end{tabular}

\section{G. Analysis of Linearization of MINLP Model}

To deal with the linearization error of voltage after applying linearization techniques, the minimum limit of voltage is set as 0.95 p.u. in order to reserve a small security margin ( 0.02 p.u.) compared with the assumed physical limit 0.93 p.u.. The linearization errors of voltage during the restoration horizon are shown in Fig. 3. Due to limited space, only linearization errors of phase $c$ are shown. The maximum error of three phases is 0.018 p.u. and is smaller than the preset security margin. Therefore, the voltage security of the solution with the MILP model is guaranteed.

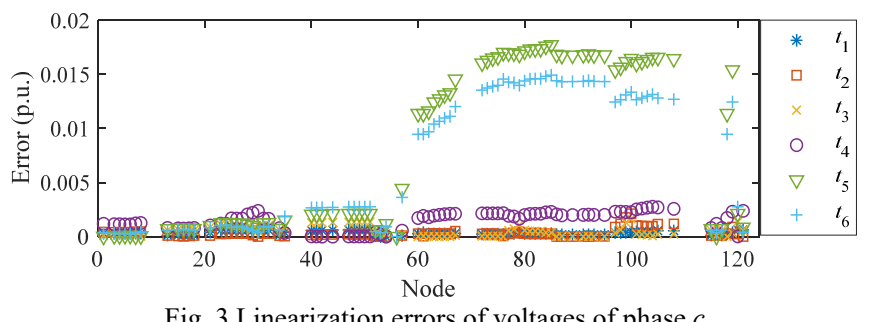

Fig. 3 Linearization errors of voltages of phase $c$

The centralized load pick-up solutions of the MINLP model and the MILP model are shown in Table X. It can be seen that two solutions have similar restoration procedures and the relative difference of the objective value is $0.19 \%$, which demonstrates that the proposed MILP model can obtain a highquality solution. Moreover, the distributed load pick-up solution obtained by the P-ADMM-based algorithm is a feasible load pick-up solution of the original MINLP model. The relative difference of the MINLP objective function value between the distributed load pick-up solution and the MINLP solution is $3.2 \%$, which indicates that the distributed load pickup solution is a high-quality solution.

TABLE X

Centralized LoAd Pick-Up SOlUtions of The MinlP AND MILP PROBLEMS

\begin{tabular}{ccccccc}
\hline \hline \multirow{2}{*}{ problems } & \multicolumn{7}{c}{ time steps } & \multirow{2}{*}{ obj. value } \\
\cline { 2 - 6 } & $t_{1}$ & $t_{2}$ & $t_{3}$ & $t_{4}$ & $t_{5}$ & \\
\hline MINLP & $31.2 \%$ & $52.4 \%$ & $73.7 \%$ & $93.5 \%$ & $100 \%$ & 298.00 \\
\hline MILP & $29.0 \%$ & $54.4 \%$ & $73.9 \%$ & $93.2 \%$ & $100 \%$ & 297.43 \\
\hline \hline
\end{tabular}

\section{CONCLUSIONS}

This paper proposes a distributed risk-limiting load restoration strategy for unbalanced DSs with networked MGs based on the CVaR and P-ADMM. By using the CVaR as the risk-limiting index, the optimal risk-limiting load restoration problem is formulated as an MINLP model that is transformed into an MILP model by applying linearization techniques. With the sub-additivity of the CVaR and P-ADMM, the MILP model is decoupled and solved in a distributed manner, realizing distributed risk-limiting strategy among MGs. The simulation results show that the proposed strategy can restore loads efficiently with a smaller risk index and can realize distributed and flexible risk management. Moreover, the proposed strategy can reduce the computation burden and is more robust against controller failures compared with the centralized strategy.

\section{REFERENCES}

[1] Y. Wang, C. Chen, J. Wang, and R. Baldick, "Research on resilience of power systems under natural disasters-A review," IEEE Trans. Power Sys., vol. 31, no. 2, pp. 1604-1613, Mar. 2016.

[2] R. R. Nejad and W. Sun, "Distributed load restoration in unbalanced active distribution systems," IEEE Trans. Smart Grid, vol. 10, no. 5, pp. 57595769, Sep. 2019.

[3] J. R. Aguero, "Applying self-healing schemes to modern power distribution systems," in 2012 IEEE Power and Energy Society General Meeting, Sand Diego, CA, Jul. 2012, pp. 1-4.

[4] M. Tsai, "Development of an object-oriented service restoration expert system with load variations," IEEE Trans. Power Syst., vol. 23, no. 1, pp. 219-225, Feb. 2008.

[5] M. R. Kleinberg, K. Miu, and H. D. Chiang, "Improving service restoration of power distribution systems through load curtailment of in-service customers," IEEE Trans. Power Syst., vol. 26, no. 3, pp. 1110-1117, Aug. 2011.

[6] A. Zidan and E. F. El-Saadany, "A cooperative multi-agent framework for self-healing mechanisms in distribution systems," IEEE Trans. Smart Grid, vol. 3, no. 3, pp. 1525-1539, Sep. 2012.

[7] S. Toune, H. Fudo, T. Genji, Y. Fukuyama, and Y. Nakanishi, "Comparative study of modern heuristic algorithms to service restoration in distribution systems," IEEE Trans. Power Del., vol. 17, no. 1, pp. 173181, Jan. 2002.

[8] P. L. Cavalcante, J. F. F. J. C. Lopez, M. J. Rider, A. V. Garcia, M. R. R. Malveira, L. L. Martins, and L. c. M. Direito, "Centralized self-healing scheme for electrical distribution systems," IEEE Trans. Smart Grid, vol. 7, no. 1, pp. 145-155, Jan. 2016.

[9] J. C. Lopez, J. F. Franco, M. J. Rider, and R. Romero, "Optimal restoration/maintenance switching sequence of unbalanced three-phase distribution systems," IEEE Trans. Smart Grid, vol. 9, no. 6, pp. 60586068, Nov. 2018.

[10] Y. Xu, C. Liu, K. P. Schneider, F. K. Tuffner, and D. T. Ton, "Micro-grids for service restoration to critical load in a resilient distribution system," IEEE Trans. Smart Grid, vol. 9, no. 1, pp. 426-437, Jan. 2018.

[11]H. Gao, Y. Chen, Y. Xu, and C. Liu, "Resilience-oriented critical load restoration using micro-grids in distribution systems," IEEE Trans. Smart Grid, vol. 7, no. 6, pp. 2837-2848, Nov. 2016.

[12] C. Chen, J. Wang, F. Qiu, and D. Zhao, "Resilient distribution system by micro-grids formation after natural disasters," IEEE Trans. Smart Grid, vol. 7, no. 2, pp. 958-966, Mar. 2016.

[13] B. Chen, C. Chen, J. Wang, and K. L. Butler-Purry, "Multi-time step service restoration for advanced distribution systems and micro-grids," IEEE Trans. Smart Grid, vol. 9, no. 6, pp. 6793-6805, Nov. 2018.

[14]B. Chen, C. Chen, J. Wang and K. L. Butler-Purry, "Sequential service restoration for unbalanced distribution systems and micro-grids," IEEE Trans. Power Syst., vol. 33, no. 2, pp. 1507-1520, Mar. 2018.

[15]Z. Wang and J. Wang, "Self-healing resilient distribution systems based on sectionalization into micro-grids," IEEE Trans. Power Syst., vol. 30, no. 6, pp. 3139-3149, Nov. 2015.

[16] X. Wu, S. Shi, X. Wang, C. Duan, T. Ding, and F. Li, "Optimal black start strategy for micro-grids considering the uncertainty using a data-driven chance constrained approach," IET Gener. Transm. Distrib., vol. 13, no. 11, pp. 2236-2248, Jun. 2019.

[17]Z. Wang, C. Shen, Y. Xu, F. Liu, X. Wu, and C. Liu, "Risk-limiting load restoration for resilience enhancement with intermittent energy resources," IEEE Trans. Smart Grid, vol. 10, no. 3, pp. 2507-2522, May 2019.

[18]M. N. Alam, S. Chakrabarti, and A. Ghosh, "Networked Micro-grids: State-of-the-art and future perspectives," IEEE Trans. Ind. Inf., vol. 15, no. 3, pp. 1238-1250, Nov. 2018.

[19]S. Boyd, N. Parikh, E. Chu, B. Peleato, and J. Eckstein, "Distributed optimization and statistical learning via the alternating direction method of multipliers," Foundations and Trends in Machine Learning, vol. 3, no. 1, pp. 1-122, Jan. 2010.

[20]Q. Peng and S. H. Low, "Distributed optimal power flow algorithm for radial network, I: Balanced single phase case," IEEE Trans. Smart Grid, vol. 9, no. 1, pp. 111-121, Jan. 2018. 
[21] Y. Wang, L. Wu, and S. Wang, "A fully-decentralized consensus-based ADMM approach for DC-OPF with demand response," IEEE Trans. Smart Grid, vol.8, no. 6, pp. 2637-2647, Nov. 2017.

[22] S. Huang, Q. Wu, Y. Gao, X. Chen, B. Zhou, and C. Li, "Distributed voltage control based on ADMM for large-scale wind farm cluster connected to VSC-HVDC," IEEE Trans. Sustain. Energy, in press.

[23] X. Liu, T. Zhao, S. Yao, C. B. Soh, and P. Wang, "Distributed Operation Management of Battery Swapping-Charging Systems," IEEE Trans. Smart Grid, vol. 10, no. 5, pp. 5320-5333, Sept. 2019.

[24]R. Takapoui, N. Moehle, S. Boyd, and A. Bemporad, "A simple effective heuristic for embedded mixed-integer quadratic programming," in Proc. American Control Conf., Boston, Massachusetts, 2016, pp. 5617-5625.

[25] A. Alborz and M. C. Rotkowitz, "Improving ADMM-based optimization of mixed integer objectives," in Annual Conf. on Information Sciences and Systems, Baltimore, MD, USA, May 2017.

[26]Q. Zhang, K. Dehghanpour, and Z. Wang, "Distributed CVR in Unbalanced Distribution Systems With PV Penetration," IEEE Trans. Smart Grid, vol. 10, no. 5, pp. 5308-5319, Sept. 2019.

[27]Z. Xu, Z. Hu, Y. Song, and J. Wang, "Risk-averse optimal bidding strategy for demand-side resource aggregators in day-ahead electricity markets under uncertainty," IEEE Trans. Smart Grid, vol. 8, no. 1, pp. 96-105, Sep. 2015.

[28] J. López, J. Contreras, and J. R. S. Mantovani, "Reactive power planning under conditional-value-at-risk assessment using chance-constrained optimization," IET Gener. Transm. Distrib., vol. 9, no. 3, pp. 231-240, Feb. 2015.

[29] M. Asensio and J. Contreras, "Stochastic unit commitment in isolated systems with renewable penetration under CVaR assessment," IEEE Trans. Smart Grid, vol. 7, no. 3, pp. 1356-1367, May 2016.

[30]J. Zhao and H. Wang, "Optimization of uncertainty load restoration strategy based on assessment of conditional value-at-risk," in 2016 IEEE PES Asia-Pacific Power and Energy Engineering Conf., Xi' an, China, Dec. 2016.

[31] J. Zhao, Y. Liu, H. Wang, and Q. Wu, "Receding horizon load restoration for coupled transmission and distribution system considering load-source uncertainty," Int. J. Elect. Power and Energy Syst., vol. 116, pp. 105517, Mar. 2020.

[32] C. L. Moreira, F. O. Resende, and J. A. P. Lopes, "Using low voltage microgrids for service restoration," IEEE Trans. Power Syst., vol. 22, no. 1, pp. 395-403, Feb. 2007.

[33] M. C. Bozchalui and R. Sharma, "Optimal operation of energy storage in distribution systems with renewable energy resources," in 2014 Clemson University Power Systems Conference, Clemson, SC, 2014, pp. 1-6.

[34] W. Wu, Z. Tian, and B. Zhang, "An exact linearization method for OLTC of transformer in branch flow model," IEEE Trans. Power Syst., vol. 32, no. 3, pp. 2475-2476, May 2017.

[35]R. T. Rockafellar and S. Uryasev, "Optimization of conditional value-atrisk," J. risk, vol. 2, pp. 21-42, 1999.

[36]IEEE Power and Energy Society (PES). (2013) Distribution test feeders: 123-bus feeder. [Online]. Available: http:// ewh.ieee.org/soc/pes/dsacom/ testfeeders/

[37] W. Hu, Y. Min, Y. Zhou, and Q. Lu, "Wind power forecasting errors modelling approach considering temporal and spatial dependence," Journal of Modern Power Systems and Clean Energy, vol. 5, no. 3, pp. 489-498, May 2017.

[38]G. Papaefthymiou and D. Kurowicka, "Using copulas for modeling stochastic dependence in power system uncertainty analysis," IEEE Trans. Power Syst., vol. 24, no. 1, pp. 40-49, Feb 2009.

[39] L. Wu, M. Shahidehpour, and T. Li, "Stochastic security-constrained unit commitment," IEEE Trans. Power Syst., vol. 22, no.2, pp. 800-811, May, 2007.

[40] J. Guo, G. Hug, and O. Tonguz, "Impact of communication delay on asynchronous distributed optimal power flow using ADMM," in 2017 IEEE International Conference on Smart Grid Communications, Dresden, Germany, Oct. 2017.

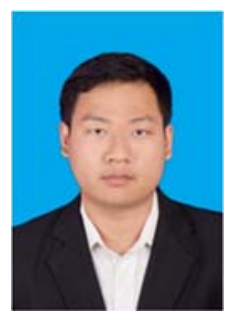

Feifan Shen received the B.Eng. degree in electrical engineering and automation from Hohai University, Nanjing, China, in 2015, and the M.Eng. degree from the Department of Electrical Engineering, Wuhan University, Wuhan, China, in 2017. He is currently pursuing the joint Ph.D. degree in the Department of Electrical Engineering, Centre of Electric Power and Energy, Technical University of Denmark, Kongens Lyngby, Denmark, and School of Electrical and Electronic Engineering, Nanyang Technological University, Singapore.

His current research interests include congestion management and self-healing for distribution networks, local flexibility market design and application, small signal stability analysis, and reactive power optimization.

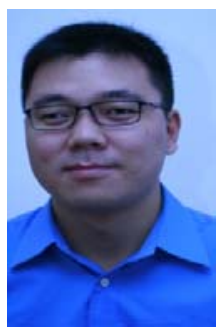

Qiuwei Wu obtained the $\mathrm{PhD}$ degree in Power System Engineering from Nanyang Technological University, Singapore, in 2009.

$\mathrm{He}$ was a senior $\mathrm{R} \& \mathrm{D}$ engineer with VESTAS Technology R\&D Singapore Pte Ltd from Mar. 2008 to Oct. 2009. He has been working at Department of Electrical Engineering, Technical University of Denmark (DTU) since Nov. 2009 (PostDoc Nov. 2009-Oct. 2010, Assistant Professor Nov. 2010-Aug. 2013, Associate Professor since Sept. 2013). He was a visiting scholar at Department of Industrial Engineering \& Operations Research (IEOR), University of California, Berkeley, from Feb. 2012 to May 2012 funded by the Danish Agency for Science, Technology and Innovation (DASTI), Denmark. He was a visiting professor named by Y. Xue, an Academician of Chinese Academy of Engineering, at Shandong University, China, from Nov. 2015 to Oct. 2017. He was a visiting scholar at the Harvard China Project, School of Engineering and Applied Sciences, Harvard University from Nov. 2017 - Oct. 2018 funded by the Otto Mønsted Fond. His research interests are operation and control of power systems with high penetration of renewables, including wind power modelling and control, active distribution networks, and operation of integrated energy systems. He is an Editor of IEEE Transactions on Smart Grid and IEEE Power Engineering Letters. He is the Deputy Editor-In-Chief of International Journal of Electrical Power and Energy Systems. He is the Regional Editor for Europe of IET Renewable Power Generation and a subject editor of IET Generation, Transmission \& Distribution.

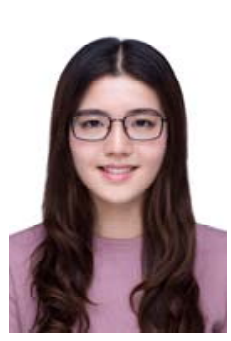

Jin Zhao received the B.Sc. degree in electrical engineering in 2015 from Shandong University, Jinan, China, where she is working toward the Ph.D. degree in electrical engineering.

She is currently a visiting student at the Department of Electrical Engineering, Technical University of Denmark (DTU). Her research interests include power system resilience, restoration, renewable energy integration, and power system optimization.

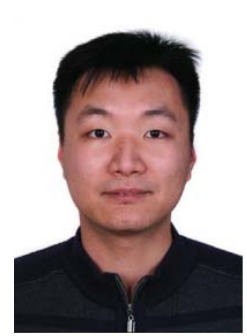

Wei Wei (M'15-SM'18) received the B.Sc. and Ph.D. degrees in electrical engineering from Tsinghua University, Beijing, China, in 2008 and 2013, respectively. Dr. Wei was a Postdoctoral Research Fellow with Tsinghua University from 2013 to 2015 . He was a Visiting Scholar with Cornell University, Ithaca, NY, USA, in 2014, and with Harvard University, Cambridge, MA, USA, in 2015. He is currently an Associate Professor with Tsinghua University. His research interests include applied optimization and energy economics 


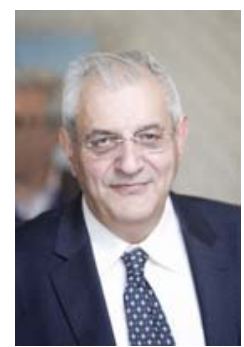

Nikos D. Hatziargyriou is full professor in Power Systems at the Elec-trical and Computer Engineering School of the National Technical Uni-versity of Athens. From 2015 to 2019 he was Chair and until 2018, CEO of the Hellenic Distribution Network Operator (HEDNO). From 2007 until 2012 he was executive Vice-Chair and Deputy CEO of the Public Power Corporation (PPC), responsible for the Transmission and Distribution Divisions. He is Fellow Member of IEEE, past Chair of the Power System Dynamic Performance Committee (PSDPC) and currently Editor in Chief of the IEEE Trans on Power Systems. He is honorary member of CIGRE and past Chair of CIGRE SC C6 "Distribution Sys-tems and Distributed Generation". He was chair and currently vice-chair of the EU Technology and Innovation Platform on Smart Networks for Energy Transition (ETIP-SNET). He has participated in more than 60 RD\&D projects funded by the EU Commission, electric utilities and manufacturers. He is author of the book "Microgrids: Architectures and Control" and of more than 250 journal publications and 500 conference proceedings papers. He is included in the 2016, 2017 and 2019 Thom-son Reuters lists of the top 1\% most cited researchers.

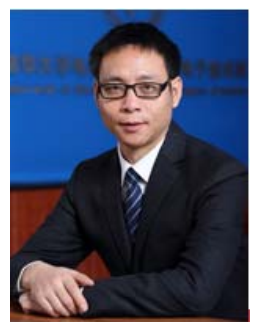

Feng Liu received the B.Sc. and Ph.D. degrees in electrical engineering from Tsinghua University, Beijing, China, in 1999 and 2004, respectively.

$\mathrm{He}$ is currently an Associate Professor with

Tsinghua University, Beijing, China. His research interests include power system analysis and control, and renewable generation. 